Manuscript Number:

Title: The sp2-iminosugar glycolipid 1-dodecylsulfonyl-5N,60oxomethylidenenojirimycin (DSO2-ONJ) as selective anti-inflammatory agent by modulation of hemeoxygenase-1 in BV.2 microglial cells and retinal explants.

Article Type: Full Length Article

Keywords: Diabetic retinopathy, inflammation, p38 MAPK, sp2-iminosugar, glycolipid, heme oxygenase-1

Corresponding Author: Dr. Ana I Arroba, PhD

Corresponding Author's Institution: IIB

First Author: Elena Alcalde-Estevez

Order of Authors: Elena Alcalde-Estevez; Ana I Arroba, PhD; Elena M Sánchez-Fernández, PhD; Carmen Ortiz-Mellet, PhD; Jose M GarcíaFernández, PhD; Laura Masgrau; Ángela M Valverde, PhD

Abstract: Neuroinflammation is an early event during diabetic retinopathy (DR) that impacts the dynamics of microglia polarization. Gliosis is a hallmark of DR and we have reported the beneficial effects of 1R-DSO-ONJ, a member of the sp2-iminosugar glycolipid (sp2-IGL) family, in targeting microglia and reducing gliosis in diabetic db/db mice. Herein, we have analyzed the effect of DSO2-ONJ, another family compound incorporating a sulfone group that better mimics the phosphate group of phosphatidylinositol ether lipid analogs (PIAs), in BV.2 microglial cells treated with bacterial lipopolysaccaride (LPS) and in retinal explants from $\mathrm{db} / \mathrm{db}$ mice. In addition to decreasing iNos and inflammasome activation, the anti-inflammatory effect of DSO2-ONJ was mediated by direct p38 $\mathrm{MAPK}$ activation. Computational docking experiments demonstrated that DSO2-ONJ binds to p38 $\mathrm{MAPK}$ at the same site where the PIAs and the alkyl phospholipid perifosine activators do, suggesting a similar mechanism of action. Moreover, treatment of microglial cells with DSO2-ONJ increased both heme-oxygenase (HO)-1 and Ill0 expression regardless the presence of LPS. In retinal explants from $\mathrm{db} / \mathrm{db} \mathrm{mice}$, DSO2-ONJ also induced $\mathrm{HO}-1$ and reduced gliosis. Since IL-10-mediated

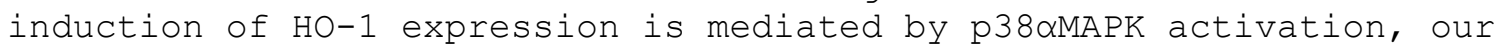
results suggest that this molecular mechanism is involved in the antiinflammatory effects of DSO2-ONJ in microglia.

Suggested Reviewers: Valentín Ceña PhD

Professor, Pharmacology, Universidad de Castilla-La Mancha. Albacete. Spain

valentin.cena@gmail.com

Professor V. Ceña is expertise in neurodegenerative diseases and apoptosis.

Evelyn Schaeffer PhD 
Professor, Immunopathologie et Chimie Thérapeutique, Université de Strasbourg-CNRS

e.schaefferlibmc-cnrs.unistra.fr

Professor E. Schaeffer is expertise in immnology interactions in health and disease

Antonio Campos-Caro PhD

Laboratory Head, Reseach Unit, Hospital Universitario Puerta del Mar.

Cádiz. Spain

antonio.campos.caro@sspa.juntadeandalucia.es

Dr. A. Campos-Caro is expertise in immunology, Molecular Biology, Cancer Research

Stella Vujosevic MD, PhD, FEBO

Head Medical Retina, Ophthalmology, University Hospital Maggiore della Carita.Padova. Italy

stella.vujosevic@gmail.com

Dr. S. Vujosevic is expertise in retinal degenerations

Luis Goya PhD

Head of the Department, Metabolism and nutrition, Insituto del Frio-CSIC. Madrid. Spain

luisgoya@if.csic.es

Dr. L. Goya is expertise in metabolism and nutrition,

Opposed Reviewers: 
Dear Editor:

Find enclosed the electronic submission of the manuscript entitled "The sp2-iminosugar glycolipid 1-dodecylsulfonyl-5N,60-oxomethylidenenojirimycin (DSO2-ONJ) as selective antiinflammatory agent by modulation of hemeoxygenase-1 in Bv2.microglial cells and retinal explants" to be considered for publication in Food and Chemical Toxicology.

Neuroinflammation has been described as an early event in the progression of diabetic retinopathy (DR). Associated with inflammation, gliosis is a hallamark of DR and recently we have reported the beneficial effects of the bicyclic compound $(1 R)-1$-dodecylsulfinyl-5N,6Ooxomethylidenenojirimycin (1R-DSO-ONJ), a member of the $\mathrm{sp}^{2}$-iminosugar glycolipid $\left(\mathrm{sp}^{2}-\right.$ IGL) family, in targeting microglia, the immune resident cells of the retina, and reducing reactive gliosis in diabetic $d b / d b$ mice. In the present study we have analyzed the effect of $\mathrm{DSO}_{2}-\mathrm{ONJ}_{\text {, }}$ another compound of the family incorporating a sulfone group that better mimics the phosphate group of phosphatidylinositol ether lipid analogs (PIAs), in Bv.2 microglial cells treated with bacterial lipopolysaccaride (LPS) and in retinal explants from $d b / d b$ mice. In addition to decreasing iNOS and targeting nuclear factor kappa B (NFKB)-mediated signaling and the inflammasome, the anti-inflammatory effect of $\mathrm{DSO}_{2}-\mathrm{ONJ}$ was likely mediated by direct $\mathrm{p} 38 \alpha$ MAPK activation. We performed computational docking experiments revealing that $\mathrm{DSO}_{2}-\mathrm{ONJ}$ binds to p38 $\alpha$ MAPK at the same site where the PIAs and the alkyl phospholipid perifosine activators do, suggesting a similar mechanism of action. Importantly, treatment of $\mathrm{Bv} .2$ microglial cells with $\mathrm{DSO}_{2}-\mathrm{ONJ}$ increased both heme oxygenase (HO)-1 protein and $\mathrm{Il} 10 \mathrm{mRNA}$ levels regardless the presence of LPS. In retinal explants from $d b / d b$ mice, this compound also induced HO-1 expression, decreased iNOS levels and reduced reactive gliosis. Since IL-10mediated induction of HO-1 expression is dependent on p38 $\alpha$ MAPK activation, our results strongly suggest that this molecular mechanism is likely involved in the anti-inflammatory effects of $\mathrm{DSO}_{2}-\mathrm{ONJ}$ in microglia. Altogether, our results provide new molecular insights on the therapeutic effects of the $\mathrm{sp}^{2}$-iminosugar glycolipid $\mathrm{DSO}_{2}$-ONJ against retinal neuroinflammation during DR. 
Looking forward to the Editorial decision of the manuscript and with our thanks for reviewing this work.

Sincerely,

Ana I Arroba PhD and Ángela M. Valverde, PhD Instituto de Investigaciones Biomédicas Alberto Sols.

Consejo Superior de Investigaciones Científicas. Madrid. Spain.

Emails: aarroba@iib.uam.es; avalverde@iib.uam.es 


\section{The $\mathrm{sp}^{2}$-iminosugar glycolipid 1-dodecylsulfonyl-5N,6O-}

oxomethylidenenojirimycin ( $\left.\mathrm{DSO}_{2}-\mathrm{ONJ}\right)$ as selective anti-inflammatory agent by modulation of hemeoxygenase-1 in Bv.2 microglial cells and retinal explants

Elena Alcalde-Estévez ${ }^{1,2^{*}}$, Ana I. Arroba ${ }^{1,2^{*} \dagger}$, Elena M. Sánchez-Fernández ${ }^{3}$, Carmen Ortiz Mellet ${ }^{3}$, Jose M. García Fernández, ${ }^{4}$ Laura Masgrau, ${ }^{5,6}$ and Ángela M.

$$
\text { Valverde }{ }^{1,2 \#} \text {. }
$$

${ }^{1}$ Instituto de Investigaciones Biomédicas Alberto Sols (IIBm) (CSIC/UAM), C/ Arturo Duperier 4, 28029 Madrid, Spain. ${ }^{2}$ Centro de Investigación Biomédica en Red de Diabetes y Enfermedades Metabólicas Asociadas (CIBERdem), ISCIII, 28029 Madrid, Spain. ${ }^{3}$ Departamento de Química Orgánica, Facultad de Química, Universidad de Sevilla, Sevilla, Spain. ${ }^{4}$ Instituto de Investigaciones Químicas (IIQ), CSIC Universidad de Sevilla, Avda. Américo Vespucio 49, 41092 Sevilla, Spain. ${ }^{5}$ Institut de Biotecnologia i de Biomedicina and ${ }^{6}$ Departament de Bioquímica i Biologia Molecular, Universitat Autònoma de Barcelona, 08193 Bellaterra, Spain

*Equal contributors

Email addresses: Ana I. Arroba (anaarroba@gmail.com); Elena Alcalde-Estevez (elena_22_93@ hotmail.com); Elena Sánchez-Fernández (esanchez4@us.es); Carmen Ortíz Mellet (mellet@us.es); José María García Fernández (jogarcia@iiq.csic.es); Laura Masgrau (Laura.Masgrau@uab.cat); Ángela M. Valverde (avalverde@iib.uam.es)

\#Corresponding authors: Ana I. Arroba (aarroba@iib.uam.es) and Ángela M. Valverde (avalverde@iib.uam.es). Instituto de Investigaciones Biomédicas Alberto Sols, C/ Arturo Duperier 4, 28029 Madrid, Spain. Telephone: 3491 5854497; Fax: 3491 5854401. 
$\dagger$ Present address: Generation and Management of the Knowledge Unit. Research Support. Area of Health Management North of Cádiz. Hospital de Jerez. Ctra. De Circunvalación s/n. 11407 Jerez de la Frontera, Cádiz, Spain.

email: anai.arroba.exts@juntadeandalucia.es 


\section{ABSTRACT}

Neuroinflammation is an early event during diabetic retinopathy (DR) that impacts the dynamics of microglia polarization. Gliosis is a hallmark of DR and we have reported the beneficial effects of 1R-DSO-ONJ, a member of the $\mathrm{sp}^{2}$-iminosugar glycolipid $\left(\mathrm{sp}^{2}-\right.$ IGL) family, in targeting microglia and reducing gliosis in diabetic $d b / d b$ mice. Herein, we have analyzed the effect of $\mathrm{DSO}_{2}-\mathrm{ONJ}$, another family compound incorporating a sulfone group that better mimics the phosphate group of phosphatidylinositol ether lipid analogs (PIAs), in Bv.2 microglial cells treated with bacterial lipopolysaccaride (LPS) and in retinal explants from $d b / d b$ mice. In addition to decreasing iNOS and inflammasome activation, the anti-inflammatory effect of $\mathrm{DSO}_{2}-\mathrm{ONJ}$ was mediated by direct p38aMAPK activation. Computational docking experiments demonstrated that $\mathrm{DSO}_{2}$-ONJ binds to p38aMAPK at the same site where the PIAs and the alkyl phospholipid perifosine activators do, suggesting a similar mechanism of action. Moreover, treatment of microglial cells with $\mathrm{DSO}_{2}-\mathrm{ONJ}$ increased both hemeoxygenase (HO)-1 and $I l 10$ expression regardless the presence of LPS. In retinal explants from $d b / d b$ mice, $\mathrm{DSO}_{2}-\mathrm{ONJ}$ also induced $\mathrm{HO}-1$ and reduced gliosis. Since IL10-mediated induction of HO-1 expression is mediated by p38aMAPK activation, our results suggest that this molecular mechanism is involved in the anti-inflammatory effects of $\mathrm{DSO}_{2}-\mathrm{ONJ}$ in microglia. 


\section{The $\mathrm{sp}^{2}$-iminosugar glycolipid 1-dodecylsulfonyl-5N,6O-}

\section{oxomethylidenenojirimycin $\left(\mathrm{DSO}_{2}-\mathrm{ONJ}\right)$ as selective anti-inflammatory agent by modulation of hemeoxygenase-1 in Bv.2 microglial cells and retinal explants}

Elena Alcalde-Estévez ${ }^{1,2^{*}}$, Ana I. Arroba ${ }^{1,2^{*} \dagger}$, Elena M. Sánchez-Fernández ${ }^{3}$, Carmen Ortiz Mellet ${ }^{3}$, Jose M. García Fernández, ${ }^{4}$ Laura Masgrau, ${ }^{5,6}$ and Ángela M. Valverde ${ }^{1,2 \#}$.

${ }^{1}$ Instituto de Investigaciones Biomédicas Alberto Sols (IIBm) (CSIC/UAM), C/ Arturo Duperier 4, 28029 Madrid, Spain. ${ }^{2}$ Centro de Investigación Biomédica en Red de Diabetes y Enfermedades Metabólicas Asociadas (CIBERdem), ISCIII, 28029 Madrid, Spain. ${ }^{3}$ Departamento de Química Orgánica, Facultad de Química, Universidad de Sevilla, Sevilla, Spain. ${ }^{4}$ Instituto de Investigaciones Químicas (IIQ), CSIC Universidad de Sevilla, Avda. Américo Vespucio 49, 41092 Sevilla, Spain. ${ }^{5}$ Institut de Biotecnologia i de Biomedicina and ${ }^{6}$ Departament de Bioquímica i Biologia Molecular, Universitat Autònoma de Barcelona, 08193 Bellaterra, Spain

*Equal contributors

Email addresses: Ana I. Arroba (anaarroba@gmail.com); Elena Alcalde-Estevez (elena_22_93@hotmail.com); Elena Sánchez-Fernández (esanchez4@us.es); Carmen Ortíz Mellet (mellet@us.es); José María García Fernández (jogarcia@iiq.csic.es); Laura Masgrau (Laura.Masgrau@uab.cat); Ángela M. Valverde (avalverde@iib.uam.es) \#Corresponding authors: Ana I. Arroba (aarroba@iib.uam.es) and Ángela M. Valverde (avalverde@iib.uam.es). Instituto de Investigaciones Biomédicas Alberto Sols, C/ Arturo Duperier 4, 28029 Madrid, Spain. Telephone: 3491 5854497; Fax: 3491 5854401. 
$\dagger$ Present address: Generation and Management of the Knowledge Unit. Research Support. Area of Health Management North of Cádiz. Hospital de Jerez. Ctra. De Circunvalación s/n. 11407 Jerez de la Frontera, Cádiz, Spain. email: anai.arroba.exts@juntadeandalucia.es 


\section{ABSTRACT}

Neuroinflammation is an early event during diabetic retinopathy (DR) that impacts the dynamics of microglia polarization. Gliosis is a hallmark of DR and we have reported the beneficial effects of 1R-DSO-ONJ, a member of the $\mathrm{sp}^{2}$-iminosugar glycolipid $\left(\mathrm{sp}^{2}-\right.$ IGL) family, in targeting microglia and reducing gliosis in diabetic $d b / d b$ mice. Herein, we have analyzed the effect of $\mathrm{DSO}_{2}-\mathrm{ONJ}$, another family compound incorporating a sulfone group that better mimics the phosphate group of phosphatidylinositol ether lipid analogs (PIAs), in Bv.2 microglial cells treated with bacterial lipopolysaccaride (LPS) and in retinal explants from $d b / d b$ mice. In addition to decreasing iNOS and inflammasome activation, the anti-inflammatory effect of $\mathrm{DSO}_{2}-\mathrm{ONJ}$ was mediated by direct p38aMAPK activation. Computational docking experiments demonstrated that $\mathrm{DSO}_{2}$-ONJ binds to p38aMAPK at the same site where the PIAs and the alkyl phospholipid perifosine activators do, suggesting a similar mechanism of action. Moreover, treatment of microglial cells with $\mathrm{DSO}_{2}-\mathrm{ONJ}$ increased both hemeoxygenase (HO)-1 and $I l 10$ expression regardless the presence of LPS. In retinal explants from $d b / d b$ mice, $\mathrm{DSO}_{2}-\mathrm{ONJ}$ also induced $\mathrm{HO}-1$ and reduced gliosis. Since IL10-mediated induction of HO-1 expression is mediated by p38aMAPK activation, our results suggest that this molecular mechanism is involved in the anti-inflammatory effects of $\mathrm{DSO}_{2}$-ONJ in microglia.

KEY WORDS: Diabetic retinopathy, inflammation, p38 MAPK, sp ${ }^{2}$-iminosugar, glycolipid, heme oxygenase-1 


\section{ABBREVIATIONS:}

ARVO: Association for Research in Vision and Ophthalmology

BSA: Bovine Serum Albumin

CNS: Central Nervous System

DAPI: 4,6-diamidino-2-phenylindole

DR: Diabetic Retinopathy

$\mathrm{DSO}_{2}$-ONJ: (1R)-1-dodecylsulfonyl-5N,6O-oxomethylidenenojirimycin

EGTA: Ethylene Glycol Tetraacetic Acid

FBS: Fetal Bovine Serum

FELASA: Federation of European Laboratory Animal Science Associations

GFAP: Glial Fibrillary Acidic Protein

HO-1: Heme oxygenase-1

HRP: Horseradish peroxidase

$\mathrm{I} \kappa \mathrm{B} \alpha$ : Inhibitor of nuclear factor- $\kappa \mathrm{B}, \alpha$

IL1 $\beta$ : Interleukine $1 \beta$

IL8: Interleukine 8

IL10: Interleukine 10

iNOS: Inducible Nitric Oxide Synthase 
JNK: c-Junk N-terminal Kinase

LPS: Bacterial Lipopolysaccharide

MAPKs: Mitogen-Activated Protein Kinases

MCP-1: Monocyte chemoattractant protein-1

MMFF94: Merck Molecular Force Field 94

MKKs: Mitogen-Activated Protein Kinase Kinases

NADPH: Nicotinamide Adenine Dinucleotide Phosphate

NEDA: N-(1-naphthyl) ethylenediamine

NF- $\kappa B$ : Nuclear factor- $\kappa B$

NLRP3 inflammasome: NACHT, LRR and PYD domains-containing protein 3 inflammasome complex

Nrf2: Nuclear factor-erythroid 2-related factor 2

PDB: Protein Data Bank

PIAs: Phosphatidylinositol Ether Lipid Analogs

PVDF: Polyvinyl difluoride

qPCR: Quantitative Polymerase Chain Reaction

ROS: Reactive Oxygen Species

RT-PCR: Real Time Polymerase Chain Reaction

SEM: Standard Error of the Measurement 
SDS-PAGE: Sodium Dodecyl Sulphate-Polyacrylamide Gel Electrophoresis

$\mathrm{sp}^{2}$-IGL: $\mathrm{sp}^{2}$-Iminosugar Glycolipid

1R-DSO-ONJ: (1R)-1-dodecylsulfinyl-5N,6O-oxomethylidenenojirimycin

T2DM: Type 2 Diabetes Mellitus

TNF- $\alpha$ : Tumor Necrosis Factor- $\alpha$

\section{HIGHLIGHTS:}

1. The $\mathrm{sp}^{2}$-iminosugar glycolipid $\mathrm{DSO}_{2}$-ONJ targets microglial $\mathrm{Bv} .2$ cells and reduced LPS-mediated pro-inflammatory responses.

2. The anti-inflammatory effect of $\mathrm{DSO}_{2}-\mathrm{ONJ}$ was mediated by direct $\mathrm{p} 38 \alpha \mathrm{MAPK}$ activation.

3. $\mathrm{DSO}_{2}-\mathrm{ONJ}$ increased $\mathrm{HO}-1$ and $\mathrm{Il} 10$ in retinal explants from $d b / d b$ mice, which reduced reactive gliosis.

4. Docking experiments are consistent with $\mathrm{DSO}_{2}-\mathrm{ONJ}$ binding at a hydrophobic pocket located at the C'-lobe of $\mathrm{p} 38 \alpha$ MAPK.

5. Similarly to phosphatidylinositol ether analogs or perifosine, $\mathrm{DSO}_{2}-\mathrm{ONJ}$ likely induces autophosphorilation of p38 $\alpha$ MAPK. 


\section{INTRODUCTION}

Type 2 diabetes mellitus (T2DM) is recognized as a low grade chronic inflammatory disease in which initially the infiltration of immune cells in the adipose tissue triggers a systemic pro-inflammatory milieu that negatively interferes with the insulin action in peripheral tissues, as well as dysregulate whole body energy balance [1]. Inflammation, together with hyperglycemia are responsible for a prolonged metabolic stress that also impacts the central nervous system (CNS), leading to neuroinflammation [2].

Recently, neuroinflammation has been described as an early event in the progression of diabetic retinopathy (DR), a major diabetic complication and one of the leading causes of blindness worldwide [3]. Similar to other neurodegenerative diseases, DR exhibits an inflammatory component associated with changes in retinal expression of inflammatory mediators, which occurs in concert with functional changes in retinal permeability and cellular viability [4]. DR-associated retinal diseases such as proliferative diabetic retinopathy and diabetic macular edema are often accompanied by activation of microglial cells that constitute the immune resident population in the retina $[5,6]$. In the $d b / d b$ mouse model that recapitulates the progression of DR [7], we have recently reported that the polarization of microglia is a dynamic process that switches from the anti-inflammatory M2 stage that was increased adaptively in early diabetic retinas attempting to maintain retinal homeostasis to the pro-inflammatory M1 stage in established DR that is characterized by elevations in pro-inflammatory cytokines and the activation of the inflammasome system [8]. In this regard, TNF $\alpha$, IL1 $\beta$, IL-8 and MCP-1 have been found elevated in the vitreous of diabetic patients [9, 10]. Thus, targeting microglia by switching its polarization towards the M2 stage is a therapeutic strategy to delay and/or prevent the deterioration of visual function in diabetic patients. 
Oxidative stress, which is induced by the increased accumulation of reactive oxygen species (ROS) and/or decreased anti-oxidant capacity, has an important role in the pathogenesis of DR [11]. Elevations in ROS in the diabetic retina in humans have been confirmed by several groups $[12,13]$ as well as increases in the expression and/or activity of the NADPH oxidases Nox2 and Nox4 that constitute one of the major sources of ROS in the retina [14].

Heme oxygenase-1 (HO-1), encoded by HMOX1 gene in humans or Hmoxl gene in rodents, is an anti-oxidant enzyme regulated by the transcription factor nuclear factorerythroid 2-related factor 2 (Nrf2), a master regulator of the cellular anti-oxidant responses [15]. On the other hand, it has been reported that overexpression of HO-1 in the retina was able to restore the visual function in diabetic animal models [16], suggesting that $\mathrm{HO}-1$ represents an important adaptive mechanism of the retinal tissue for moderating the severity of cell damage produced by oxidative stress. Regarding oxidative stress in the retina of $d b / d b$ mice, both HO-1 expression and activation of Nox 4 have been found increased in the retinas at the age of 8 weeks, while decreased at 20 weeks compared to their age-matched controls [17], strongly suggesting that during the early stages of DR the retina has anti-oxidant sensors that protect against the oxidative component of the diabetic milieu. Pharmacological induction of HO-1 is therefore a promising approach for therapeutic intervention in DR conditions.

Activation of mitogen-activated protein kinases (MAPKs), such as the p38 $\alpha$ MAPK, plays a central role for the induction of HO-1 gene expression [18]. Accordingly, lipidbased activators of p38 $\alpha$ MAPK, which include the phosphatidylinositol ether lipid analogs (PIAs) and the alkyl phospholipid perifosine, have demonstrated a major antiinflammatory potential $[19,20]$. However, side effects associated to hemotoxicity, 
cardiotoxicity, gastrointestinal toxicity and alteration of the insulin/glucose metabolism may represent a limitation for clinical translation [21, 22]. Thus, the identification and characterization of compounds featuring a better pharmacological profile that induce HO-1 in a cell-specific and cell context-specific manner remains a current priority.

The World Health Organization (WHO) has estimated that $80 \%$ of the world inhabitants utilized traditional medicine for their primary health care needs and the majority of this therapy requires the use of herbal extracts and their active components. Various medicinal plant bioactive extracts and their identified/isolated active constituents have shown a variety of medicinal pharmacological properties against chronic diseases with an inflammatory component such as T2DM [23, 24]. Iminosugar glycosyl hydrolase inhibitors such as 1-deoxynojimycin $[25,26]$ and castanospermine, bear strong potential in therapies such as cancer, viral infections [27, 28], tuberculosis [29], diabetes and glycosphingolipid storage disorders [30, 31]. We have recently reported the beneficial effects of the bicyclic compound (1R)-1-dodecylsulfinyl-5N,6Ooxomethylidenenojirimycin (1R-DSO-ONJ), a member of the $\mathrm{sp}^{2}$-iminosugar glycolipid $\left(\mathrm{sp}^{2}\right.$-IGL) family, in reducing reactive gliosis in retinal explants from $d b / d b$ mice by increasing the expression of the anti-inflammatory M2 marker arginase-1 [8]. At the molecular level, 1R-DSO-ONJ ameliorated the NFKB-mediated pro-inflammatory signaling cascade triggered by LPS in Bv.2 microglial cells, resulting in decreased iNOS expression and pro-inflammatory cytokines. Initially, the biological effects of $\mathrm{sp}^{2}-$ iminosugars were assumed to be the result of their interaction with glycosidases, since several representatives have been shown to behave as selective inhibitors or activators of these enzymes $[32,33]$. Notwithstanding, neither the anti-inflammatory activity of 1R-DSO-ONJ nor the anti-proliferative, anti-metastatic and anti-parasitic activities displayed by other $\mathrm{sp}^{2}$-IGLs could be correlated with glycosidase inhibition $[34,35]$. 
Instead, this range of activities is very much reminiscent of that displayed by PIAs, suggesting that $\mathrm{sp}^{2}$-IGLs and PIAs share the same kinase targets. If this hypothesis is correct, replacement of the sulfinyl group in 1R-DSO-ONJ by a sulfonyl group (1dodecylsulfonyl-5N,6O-oxomethylidenenojirimycin; $\mathrm{DSO}_{2}-\mathrm{ONJ}$, Figure 1A), a closer neutral isoster of the phosphate group in PIAs, might lead to a better candidate for DR treatment. As a proof of concept, here we report our results on the evaluation of the effect of $\mathrm{DSO}_{2}$-ONJ in Bv.2 microglial cells and retinal explants of $d b / d b$ mice in the context of inflammation and oxidative stress associated to DR. The data are consistent with the activation of $\mathrm{p} 38 \alpha$ MAPK leading to the enhancement of HO-1 expression. Computational docking experiments further support that $\mathrm{DSO}_{2}-\mathrm{ONJ}$ binds to p38 $\alpha$ MAPK at the same site where the PIAs and perifosine activators do, strongly suggesting a similar mechanism of action.

\section{RESEARCH DESIGN AND METHODS}

2.1. Reagents. Fetal bovine serum (FBS) and culture media were obtained from Invitrogen (Grand Island, NY, USA). Bovine serum albumin (BSA), crystal violet, bacterial lipopolysaccharide (LPS) and SB203580 were purchased from Sigma-Aldrich (St Louis, MO, USA). Bradford reagent, acrylamide, immunoblot PVDF membranes and chemiluminiscent HRP Substrate were purchased from Bio-Rad (Madrid, Spain).

2.2. Antibodies. Antibodies against caspase-1 (p10) (sc-514), iNOS (sc- 650), IкB $\alpha$ (sc371), p65-NFkB (p65) (sc-732), JNK (sc-571) and phospho-p38 $\alpha$ MAPK (Thr 180/Tyr182) (sc-17852-R) were purchased from Santa Cruz Biotechnology (Palo Alto, CA, USA). Anti-phospho JNK (Thr183/Tyr185) (\#4668) and anti-p38 a MAPK (\#9212) 
antibodies were purchased from Cell Signaling Technology (MA, USA). Anti-arginase1 (BD610708) antibody was purchased from BD Bioscience (Madrid, Spain). Anti-glial fibrillary acidic protein (GFAP) antibody (Z0334) was obtained from DAKO (Glostrup, Denmark) and anti- $\alpha$-tubulin (T-5168) antibody was from Sigma-Aldrich (St Louis, MO, USA).

2.3. Synthesis of the $\mathbf{s p}^{2}$-iminosugar glycolipid $\mathbf{D S O}_{2}$-ONJ. (1R)-1-Dodecylsulfonyl5N,6O-oxomethylidenenojirimycin (referred to as $\mathrm{DSO}_{2}$-ONJ, Fig. 1A) was synthesized from $(1 R)$-1,2,3,4-tetra- $O$-acetyl-5N,6O-oxomethylidenenojirimycin [36] by $S$ glycosylation with 1-dodecanethiol, oxidation of the resulting sulphide with $m$ chloroperbezoic acid and final deacetylation, following the procedures previously reported [35]. $\mathrm{DSO}_{2}-\mathrm{ONJ}$ was dissolved in DMSO (50\%) before storage at $-20^{\circ} \mathrm{C}$.

2.4. Cell culture. Mouse microglia Bv.2 cell line was supplied by Dr. M. L. Nieto (IBGM, Spain). Bv.2 cells were cultured at $37^{\circ} \mathrm{C}$ in a humidified atmosphere with $5 \%$ $\mathrm{CO}_{2}$ in RPMI supplemented with $10 \%$ (v/v) heat-inactivated FBS, $1 \%$ (v/v) penicillin/streptomycin (Sigma) and $2 \mathrm{mM}$ L-glutamine (Gibco, Carlsbad, California, USA). Cells were grown up to $70 \%$ confluence and then washed twice with PBS and further cultured in serum-free medium and stimulated with LPS $(200 \mathrm{ng} / \mathrm{ml})$ with or without $\mathrm{DSO}_{2}-\mathrm{ONJ}(1,1050 \mu \mathrm{M})$ for several time-periods.

2.5. Retinal explants. Ex vivo assays were performed with retinas from 8-week-old male $d b /+$ and $d b / d b$ mice in the $\mathrm{C} 57 \mathrm{BL} / \mathrm{Ks}$ J genetic background purchased from Charles River Laboratories (Charles River, Barcelona, Spain). Mice were maintained in light/dark (12-hours light/12-hours dark), temperature $\left(22^{\circ} \mathrm{C}\right)$ and humidity-controlled rooms, and fed ad libitum with free access to water. All animal experimentation followed recommendations of the Federation of European Laboratory Animal Science 
Associations (FELASA) on health monitoring in accordance with the regulations of the Association for Research in Vision and Ophthalmology (ARVO). Animals were killed by cervical dislocation and eyes were enucleated. The lens, anterior segment, vitreous body, retinal pigment epithelium and sclera were removed and the retinas were cultured in R16 medium (provided by Dr. P.A. Ekstrom, Lund University, Sweden) with no additional serum. Retinas were stimulated with $\mathrm{DSO}_{2}-\mathrm{ONJ}$ as indicated in the figure legends.

2.6. Analysis of the cellular viability by crystal violet staining. After cell treatments, the medium was discarded, and the remaining viable adherent cells were stained with crystal violet $(0.2 \% \mathrm{w} / \mathrm{v}$ in $2 \%$ ethanol) for $20 \mathrm{~min}$. After this time, plates were rinsed with tap water and allowed to dry, and 1\% SDS was added to solubilize them. The absorbance of each plate was read spectrophotometrically at $560 \mathrm{~nm}$.

2.7. Analysis of Nitrites $\left(\mathrm{NO}_{2}^{-}\right)$. Levels of $\mathrm{NO}_{2}{ }^{-}$were measured by using the Griess method [37]. Briefly, nitrites turn into a pink compound in contact with an acid solution containing $1 \%$ sulphanilamide and $0.1 \% \mathrm{~N}$-(1-naphthyl) ethylenediamine (NEDA), and can be quantified by a colorimetric method at $540 \mathrm{~nm}$ in a microplate reader (Versamax Tunable Microplate reader, Molecular Devices, Sunnyvaley, CA, USA).

2.8. Immunofluorescence. Bv. 2 microglial cells were seeded on coverslips 24 h before adding LPS and/or $\mathrm{DSO}_{2}$-ONJ in serum-free medium. After treatments, cells were washed in PBS, fixed with 4\% (w/v) paraformaldehyde in PBS for $10 \mathrm{~min}$ at room temperature, washed in PBS and permeated with 0.4\% (w/v) Triton X-100 (Sigma) in PBS for 20 min. Non-specific binding was blocked in PBS containing 3\% (w/v) BSA and $0.1 \%(\mathrm{w} / \mathrm{v})$ Triton $\mathrm{X}-100$ for $2 \mathrm{~h}$ and the cells were then left overnight in a humid chamber at $4{ }^{\circ} \mathrm{C}$ with rabbit anti-p65NFkB antibody in blocking buffer. Subsequently, 
the cells were washed and incubated in darkness for $2 \mathrm{~h}$ with anti-rabbit conjugated Alexa 488 antibody (Molecular Probes, ThermoFisher Scientific, Waltham, MA, USA) The cells were then washed, stained with 4,6-diamidino-2-phenylindole (DAPI) and mounted with medium (Fluoromount G). Immunofluorescence was examined in a Nikon Eclipse 90i microscope with imaging software Nis elements (Nikon, Nikon Instruments Europe B.V.).

For immunofluorescence analysis in retinal explants, eyes were fixed in $4 \%(\mathrm{w} / \mathrm{v})$ paraformaldehyde for $24 \mathrm{~h}$ at $4{ }^{\circ} \mathrm{C}$ and infiltrated with sucrose $25 \%$ (w/v). Retinal explants were washed in TBS containing $0.1 \%(\mathrm{w} / \mathrm{v})$ BSA and $0.1 \%(\mathrm{v} / \mathrm{v})$ Triton X-100 (this buffer was used for all subsequent washes), and then blocked and permeated for 2 $\mathrm{h}$ in TBS containing 3\% (w/v) BSA and 1\% (v/v) Triton X-100. The retinal explants were then incubated overnight in a humid chamber at $4{ }^{\circ} \mathrm{C}$ with rabbit anti-GFAP antibody in blocking solution. Retinal explants were washed and incubated for $90 \mathrm{~min}$ with anti-rabbit immunoglobulin antibody conjugated to Alexa 488 (1:2000; Molecular Probes, Eugene, OR). After washing, retinal explants were mounted with medium (Fluoromount G) containing DAPI. Staining was observed with an inverted laser confocal microscope LSM710 (Carl Zeiss Microscopy GmbH, Göttingen, Germany).

2.9. Quantitative real-time PCR (qPCR) analysis. Total RNA was extracted with Trizol® reagent (Invitrogen, Madrid, Spain) and reverse transcribed using a SuperScript ${ }^{\mathrm{TM}}$ III First-Strand Synthesis System for qPCR following the manufacturer's indications (Invitrogen). qPCR was performed with an ABI 7900 sequence detector. Primer-probe sets for mouse Tnfa, Il6, Illb, Il10, Nlrp3, Nos2, arginase-1 and $18 s$ were purchased as predesigned TaqMan gene expression assays (Applied Biosystems). 
2.10. Western blot. Whole retinas or Bv.2 microglial cells were homogenized in lysis buffer containing $50 \mathrm{mM}$ Tris-HCl, $\mathrm{pH}$ 7.4, $150 \mathrm{mM} \mathrm{NaCl}, 1 \mathrm{mM} \mathrm{Na}_{3} \mathrm{VO}_{4}, 1 \mathrm{mM} \mathrm{NaF}$, $1 \mathrm{mM}$ EGTA, $15 \%(\mathrm{w} / \mathrm{v}) \mathrm{NP} 40$ and $0.25 \%(\mathrm{w} / \mathrm{v})$ sodium deoxycholate, supplemented with protease inhibitors $(10 \mu \mathrm{g} / \mathrm{ml}$ leupeptin, $10 \mu \mathrm{g} / \mathrm{ml}$ aprotinin, and $100 \mu \mathrm{g} / \mathrm{ml}$ phenylmethylsulphonyl fluoride). All debris was removed by centrifugation at $14,000 \mathrm{x}$ $\mathrm{g}$ for $10 \mathrm{~min}$ at $4{ }^{\circ} \mathrm{C}$ and protein concentration was quantified using the Bio-Rad protein assay with BSA as a standard. Equivalent amounts of protein were resolved using denaturing sodium dodecyl sulphate-polyacrylamide gel electrophoresis (SDS-PAGE), followed by transfer to PVDF membranes (Bio-Rad). Membranes were blocked using 5\% non-fat dried milk or 3\% BSA in $10 \mathrm{mM}$ Tris-HCl, $150 \mathrm{mM} \mathrm{NaCl}, \mathrm{pH} 7.5$ (TBS), and incubated overnight with several antibodies (1:2000 unless otherwise stated) in chemiluminescence reagent (Bio-Rad).

2.11. Statistical analysis. Densitometry of the Western blots was performed using the ImageJ program. Values in all graphs represented the mean \pm SEM. Statistical tests were performed using SPSS 21.0 for Windows (SPSS Inc. IBM, Armonk, NY, USA). Data were analyzed by one-way ANOVA followed by Bonferroni t-test or by paired ttest when comparisons were among two groups. Differences were considered significant at $*+\# \mathrm{p} \leq 0.05$

2.12. Docking experiments. Protein-ligand docking calculations were performed with the AutoDock4 program [38]. For the $\mathrm{DSO}_{2}-\mathrm{ONJ}$ docking experiments, two slightly different sets of coordinates corresponding to the p38 $\alpha$ MAPK receptor were used: those from p38 $\alpha$ MAPK in complex with PIA23 (PDB ID 4E6A) and those from the p38 $\alpha$ MAPK:perifosine complex (PDB ID 4E6C) [39]. The calculations predict a better binding of $\mathrm{DSO}_{2}-\mathrm{ONJ}$ to the 4E6A structure (Supplementary Figure 2) and, thus, these 
will be the results presented in the Results section; the ones corresponding to the 4E6C protein coordinates are included in the Supplementary Information (Supplementary Figure 3). The initial coordinates of the $\mathrm{DSO}_{2}-\mathrm{ONJ}$ ligand were built with the Chem3D program, assuming a ${ }^{4} C_{1}$ conformation for the six-membered ring. A MMFF94 energy minimization in a continuum solvent environment $(\varepsilon=78)$ was used to optimize the $\mathrm{DSO}_{2}-\mathrm{ONJ}$ structure. For the protein, hydrogen atoms were added and the protonation states of the ionizable residues were assigned with PROPKA through the PDB2PQR server [40]. For the docking calculations, the volume for exploration was defined by a grid box centred at a point in the middle of the hydrophobic cavity $(x=6.5, y=2.0, z=$ -2.2, according to PDB ID 4E6A coordinates), with $86 \times 102 \times 112$ points and a grid spacing of $0.253 \AA$ A. Gasteiger charges were used. The docking calculations were carried out using the Lamarckian genetic algorithm, with rigid side chains for the receptor and randomly changing the torsion angles (15 rotable bonds for $\mathrm{DSO}_{2}-\mathrm{ONJ}$ ) and overall orientation of the ligand. 20 solutions were generated for each ligand. For the genetic algorithm, the recommended default values were used for all the parameters except for the number of energy evaluations, which was set to a larger value $\left(2.5 \times 10^{6}\right)$ to better account for the large dimensionality of the systems under study. For comparison, docking calculations of perifosine (which presents a similar number of rotable bonds, 19) to the 4E6C protein structure were also conducted. The same protocol was followed, except for the fact that the coordinates of perifosine were taken from the PDB ID 4E6C, when available, and the ones for the missing atoms were built with Chem3D. The corresponding results are given in the Supplementary Information (Supplementary Figure 4). Molecular representations in figures were generated with the USCF Chimera package [41]. 


\section{RESULTS}

\subsection{Effect of the $\mathrm{sp}^{2}$-iminosugar glycolipid $\mathrm{DSO}_{2}-\mathrm{ONJ}$ on the cellular viability and the expression of iNOS in LPS-stimulated Bv.2 microglial cells.}

We first tested the viability of microglia Bv.2 cells when exposed to various concentrations of the $\mathrm{sp}^{2}$-iminosugar glycolipid ( $\mathrm{sp}^{2}$-IGL) derivative 1-dodecylsulfonyl$5 \mathrm{~N}, 6 \mathrm{O}$-oxomethylidenenojirimycin (referred to as $\mathrm{DSO}_{2}-\mathrm{ONJ}$, Figure $1 \mathrm{~A}$ ) and found that this effect was dose-dependent, being reduced by $75 \%$ at $50 \mu \mathrm{M}$ concentration of $\mathrm{DSO}_{2}$-ONJ (Figure 1B). Based on these results, we used the doses of 1 and $10 \mu \mathrm{M}$ for further experiments.
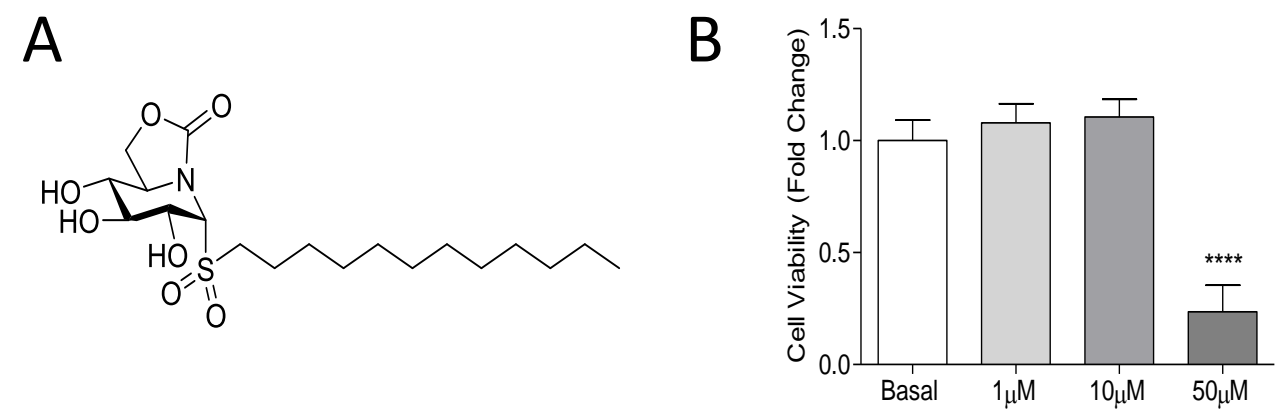

C

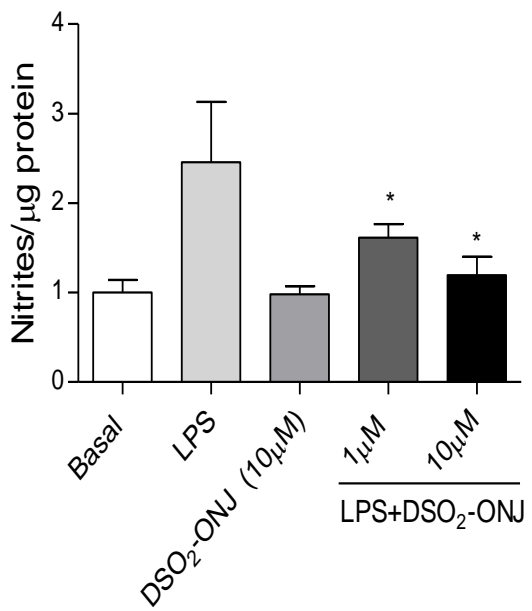

D

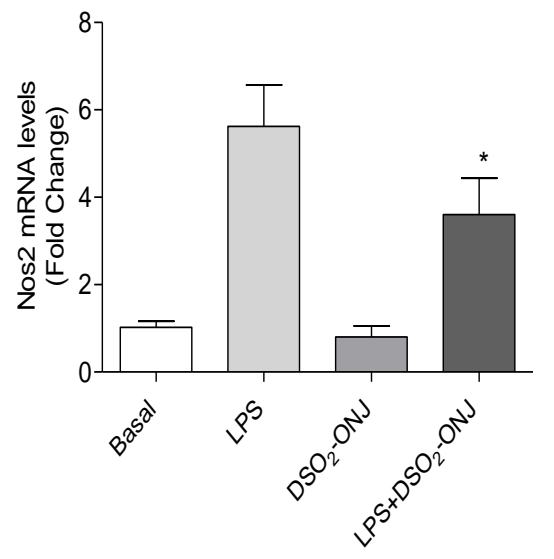


$E$
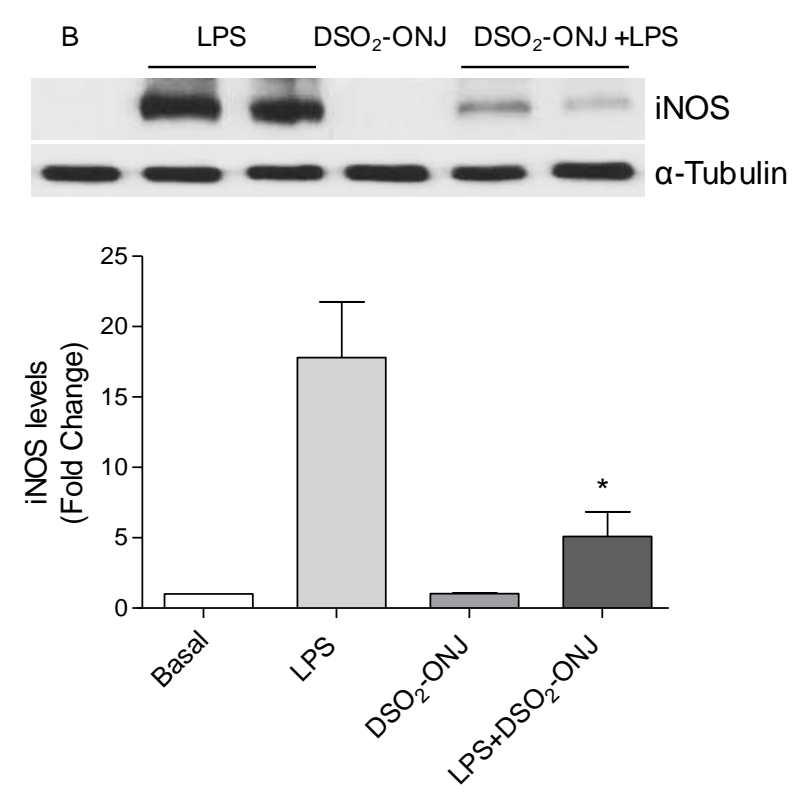

Figure 1. Effect of $\mathrm{DSO}_{2}$-ONJ on the cellular viability and the expression of iNOS in LPS-stimulated Bv.2 microglial cells. Bv.2 microglial cells were stimulated with 200

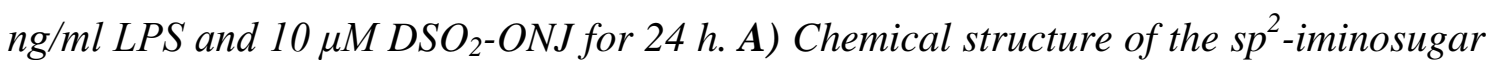
glycolipid 1-dodecylsulfonyl-5N,6O-oxomethylidenenojirimycin, referred as $\mathrm{DSO}_{2}-\mathrm{ONJ}$ in the figures. Bv.2 microglial cells were treated for $24 \mathrm{~h}$ with $\mathrm{DSO}_{2}-\mathrm{ONJ}(1-50 \mu \mathrm{M})$. B) Viability was determined by crystal violet staining. Colorimetric quantification was performed, and the results are mean \pm S.E.M. The fold change relative to Basal condition is shown. ${ }^{*} p \leq 0.05$ vs LPS treatment (two-way ANOVA followed by Bonferroni t-test. C) Bv.2 microglial cells were treated for $24 \mathrm{~h}$ with LPS (200 $\mathrm{ng} / \mathrm{ml})$ or LPS plus $\mathrm{DSO}_{2}$-ONJ (1-10 $\left.\mu \mathrm{M}\right)$. Nitrites accumulation was analyzed and related to basal levels. Colorimetric quantification was performed, and the results are mean \pm S.E.M. The fold change relative to Basal condition is shown. ${ }^{*} p \leq 0.05$ vs LPS treatment (two-way ANOVA followed by Bonferroni t-test. D) mRNA levels of Nos 2 were determined by RTPCR. The results are means \pm S.E.M. ( $n=5$ independent experiments). The fold change relative to Basal condition is shown. ${ }^{*} p \leq 0.05$ vs LPS treatment (two-way ANOVA followed by Bonferroni t-test. E) Protein extracts were analyzed by Western blot with the corresponding antibodies against iNOS and $\alpha$-Tubulin as loading control. Representative autoradiograms are shown ( $n=5$ independent experiments). Blots were quantified by performing scanning densitometry, and the results are mean \pm S.E.M. The 
fold change relative to Basal condition is shown. ${ }^{*} p \leq 0.05$ vs LPS treatment (two-way ANOVA followed by Bonferroni t-test).

In order to get more insights on the effect of $\mathrm{DSO}_{2}-\mathrm{ONJ}$ in microglia, Bv.2 cells were stimulated with LPS, a M1 stimulus, in the absence or presence of $\mathrm{DSO}_{2}-\mathrm{ONJ}$. Among other effects, LPS induces a pro-inflammatory environment similar to that found in the obesity-related diabetic context [42]. Bv.2 microglial cells were cultured for $24 \mathrm{~h}$ in the presence of LPS $(200 \mathrm{ng} / \mathrm{ml})$ or $\mathrm{DSO}_{2}-\mathrm{ONJ}(1-10 \mu \mathrm{M})$, and the combination of both $\left(\mathrm{LPS}+\mathrm{DSO}_{2}-\mathrm{ONJ}\right)$. As shown in Figure $1 \mathrm{C}$, the elevation of nitrites in the culture medium induced by LPS treatment was significantly reduced by the co-treatment with $\mathrm{DSO}_{2}-\mathrm{ONJ}$ in a dose-dependent manner. Likewise, mRNA levels of Nos 2 and protein level of iNOS, which were elevated in LPS-treated Bv.2 microglial cells, significantly decreased in the presence of $\mathrm{DSO}_{2}-\mathrm{ONJ}$ (Figure 1D).

\subsection{LPS-treated Bv.2 microglial cells induced a pro-inflammatory response and activation of the inflammasome complex that was reduced by $\mathrm{DSO}_{2}$-ONJ.}

The effect of $\mathrm{DSO}_{2}$-ONJ in the mRNA levels of pro- and anti-inflammatory cytokines in Bv.2 microglial cells was next analyzed. LPS induced a significant increase in Tnfa, $I l 1 b$ and $I l 6$ mRNA that was ameliorated by the co-treatment with $\mathrm{DSO}_{2}$-ONJ (Figure 2A). As in LPS-stimulated microglia IL1 $\beta$ is processed via caspase-1 through the NACHT, LRR and PYD domains-containing protein 3 (NLRP3) inflammasome complex [28], we explored if the anti-inflammatory effect of $\mathrm{DSO}_{2}-\mathrm{ONJ}$ was dependent on the inflammasome. The mRNA levels of Nlrp3 showed a significant increase after LPS stimulation, but were markedly reduced upon treatment with $\mathrm{DSO}_{2}-\mathrm{ONJ}$ (Figure 2B). As shown in Figure 2C, the cleavage of pro-caspase-1 in response to LPS was 
totally abolished in Bv.2 microglial cells treated with $\mathrm{DSO}_{2}-\mathrm{ONJ}$. Also, the effect of this compound in the inflammasome was assessed by the significant reduction in the cleavage of pro-IL1 $\beta$ into its active (processed) form which was induced by LPS stimulation (Figure 2D).

A
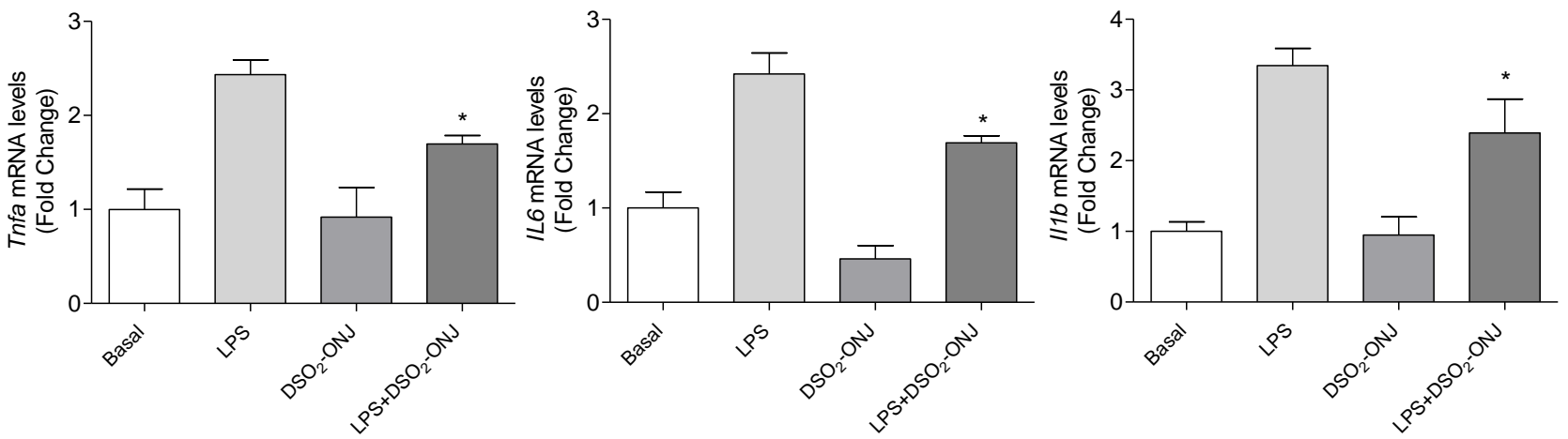

B
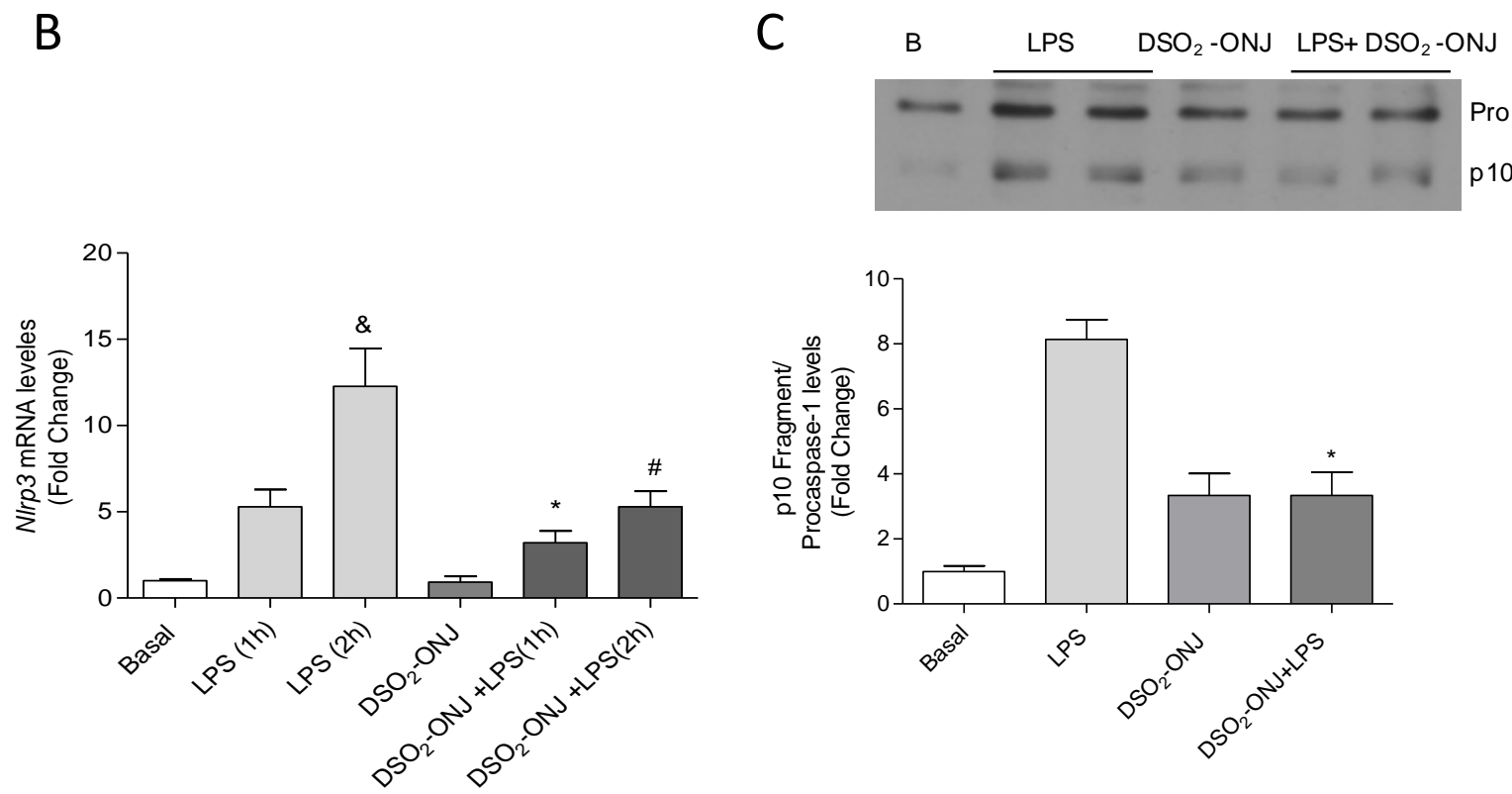
D

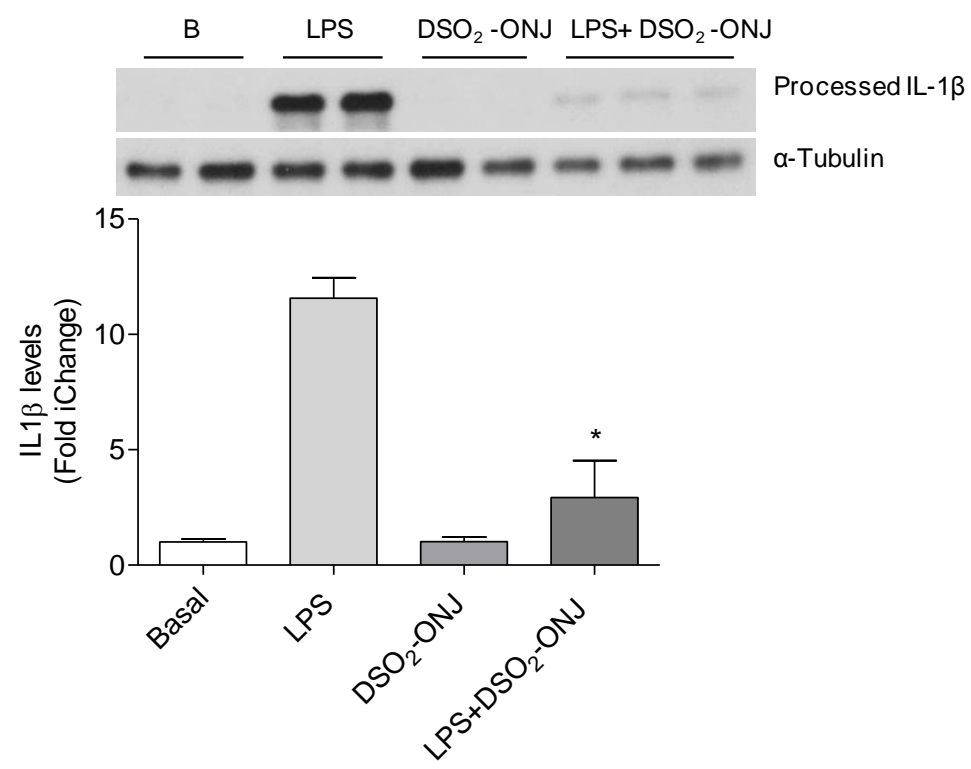

Figure 2. Protective effects of $\mathrm{DSO}_{2}-\mathrm{ONJ}$ against LPS-mediated activation of the inflammasome and elevation of pro-inflammatory cytokines mRNA levels in Bv.2 microglial cells. Bv.2 microglial cells were stimulated with $200 \mathrm{ng} / \mathrm{ml}$ LPS and $10 \mu \mathrm{M}$ $D_{S O}$-ONJ for 24 h. A) Tnfa, Illb, and Il6 mRNA levels were determined by RT-PCR. B) Nlrp3 mRNA levels were determined by RT-PCR. The results are mean \pm S.E.M. ( $n=5$ independent experiments). The fold change relative to Basal condition is shown. ${ }^{*} p \leq 0.05$ vs LPS treatment (two-way ANOVA followed by Bonferroni $t$-test). Protein extracts were analyzed by Western blot with antibodies against caspase-1 $(\boldsymbol{C})$ and $I L-1 \beta$ (D). $\alpha$-Tubulin was used as a loading control. Representative autoradiograms are shown ( $n=5$ independent experiments). Blots were quantified by performing scanning densitometry, and the results are mean \pm S.E.M. The fold change relative to Basal condition is shown. ${ }^{*} p \leq 0.05$ vs LPS treatment (two-way ANOVA followed by Bonferroni t-test).

In order to determine the molecular mechanisms involved in the anti-inflammatory effect exerted by $\mathrm{DSO}_{2}-\mathrm{ONJ}$, we examined MAPKs and $\mathrm{NF \kappa B}$-mediated signaling pathways. Treatment of Bv.2 microglial cells with LPS rapidly activated MAPKs by 
inducing the phosphorylation of JNK and p38 $\alpha$ MAPK and induced I $\mathrm{B}$ degradation; maximal effect being elicited after $30 \mathrm{~min}$ (Figure $3 \mathrm{~A}$ ). Co-treatment with $\mathrm{DSO}_{2}-\mathrm{ONJ}$ prevented LPS-mediated $\mathrm{I} \kappa \mathrm{B} \alpha$ degradation (Figure $3 \mathrm{~A}$ ), as well as the nuclear translocation of p65-NF- $\mathrm{BB}$ (Figure $3 \mathrm{~B}$ ). However, $\mathrm{DSO}_{2}-\mathrm{ONJ}$ failed to decrease the phosphorylation of JNK and p38 $\alpha$ MAPK (Figure 3A). On the contrary, co-treatment with LPS and $\mathrm{DSO}_{2}-\mathrm{ONJ}$ led to significantly higher levels of phosphorylation of both kinases as compared with the treatment with LPS alone.

\section{A}
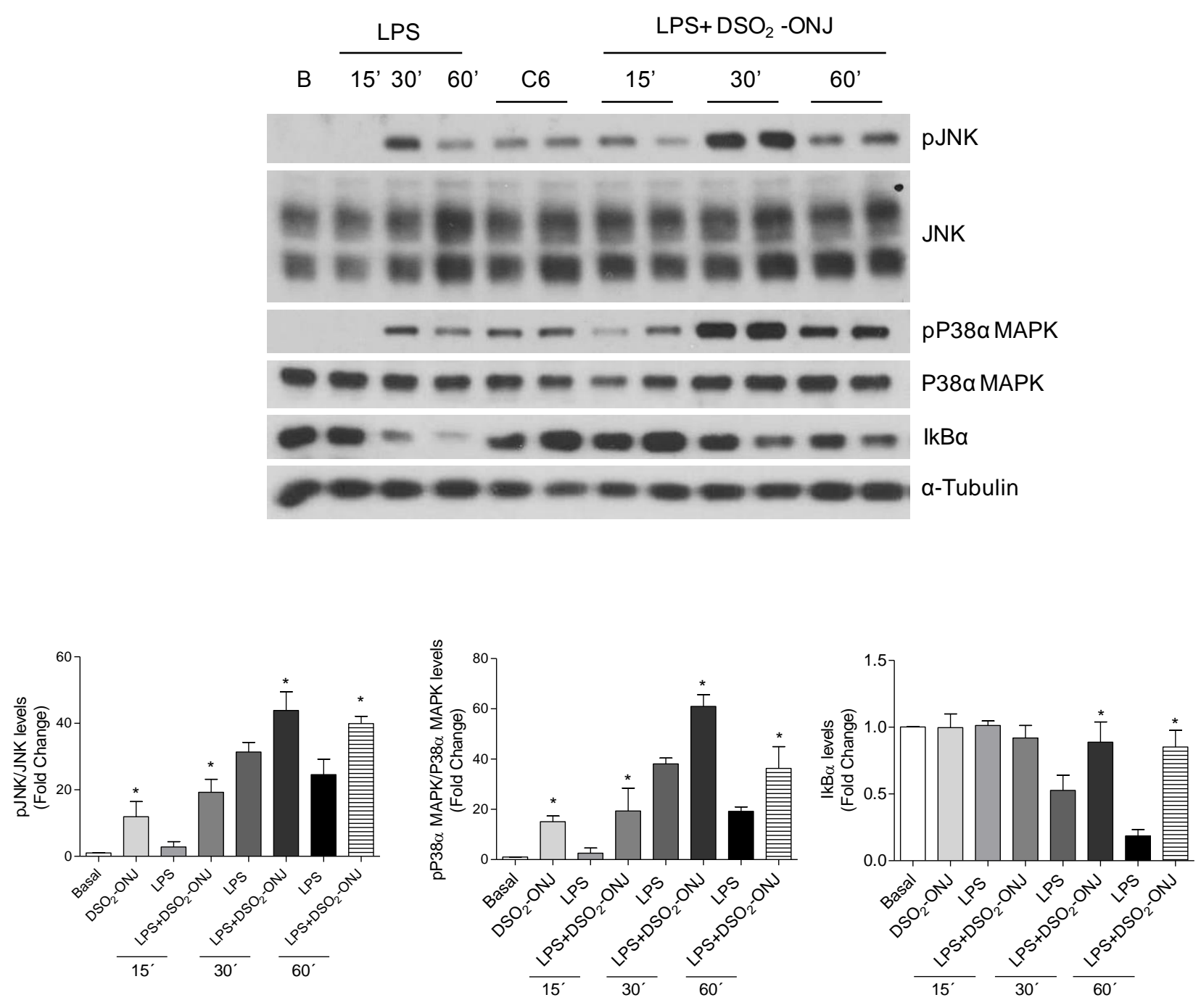
B

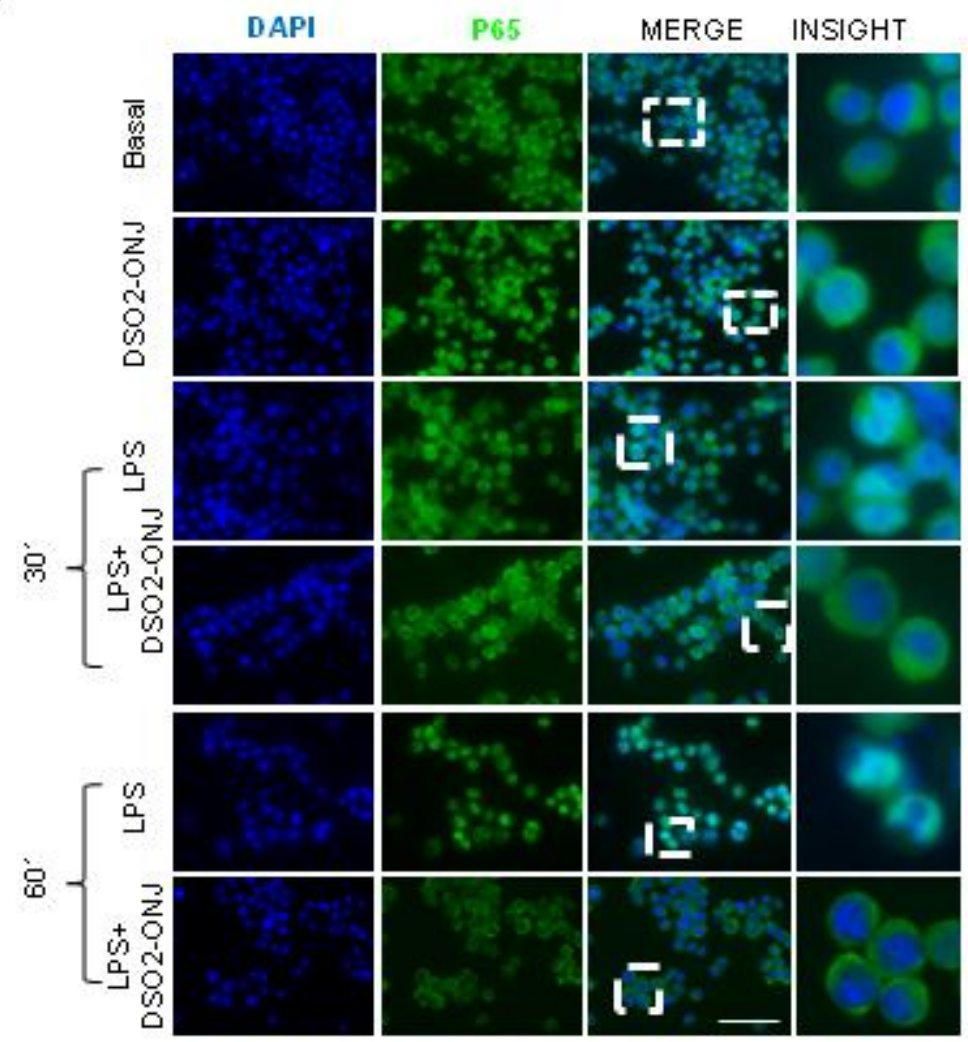

Figure 3. Effects of $\mathrm{DSO}_{2}-\mathrm{ONJ}$ in the activation of MAPKs-mediated signaling in LPS-stimulated Bv.2 microglial cells. Bv.2 microglial cells were stimulated with 200

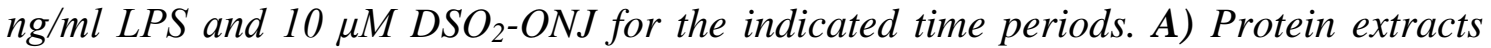
were separated by SDS-PAGE and analyzed by Western blot with antibodies against

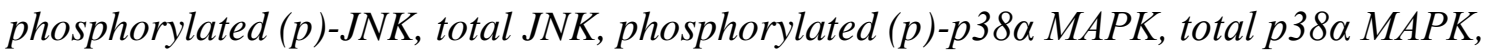
$I \kappa B \alpha$ and $\alpha$-Tubulin. Representative autoradiograms are shown ( $n=5$ independent experiments). Blots were quantified by performing scanning densitometry and the results are mean \pm S.E.M. The ratio between the indicated proteins and the fold change relative to Basal condition is shown. ${ }^{*} p \leq 0.05$ vs LPS treatment (two-way ANOVA followed by Bonferroni t-test). B) Confocal immunofluorescence assessment of nuclear translocation of p65-NFкB in Bv.2 microglial cells following stimulation with LPS in the absence or presence of $\mathrm{DSO}_{2}-\mathrm{ONJ}$. Activation of p65-NF $\kappa$ B nuclear translocation was defined by an increase in immunofluorescence of p65-NF $\mathrm{B}$ (green channel) in the nuclear regions. Nuclear regions of Bv.2 microglial cells were determined by counterstaining of nuclear DNA with DAPI (blue channel). Nuclear localization of p65- 
$N F \kappa B$ appears in LPS treatment at $30 \mathrm{~min}$ and $60 \mathrm{~min}$, but not in Bv.2 microglial cells preincubated with $10 \mu \mathrm{M} \mathrm{DSO}{ }_{2}-\mathrm{ONJ}$.

To analyze the direct effect of $\mathrm{DSO}_{2}-\mathrm{ONJ}$ in the phosphorylation of $\mathrm{JNK}$ and $\mathrm{p} 38 \alpha$ MAPK, we performed a time-course experiment. Figure 4 shows that $\mathrm{DSO}_{2}-\mathrm{ONJ}$ induced the phosphorylation of JNK and p38 $\alpha$ MAPK, with a maximal effect being elicited at 60 min. Supplementary Figure 1 shows that $\mathrm{DSO}_{2}-\mathrm{ONJ}$-induced p38 $\alpha$ MAPK phosphorylation was totally abolished by the inhibitor SB203580.
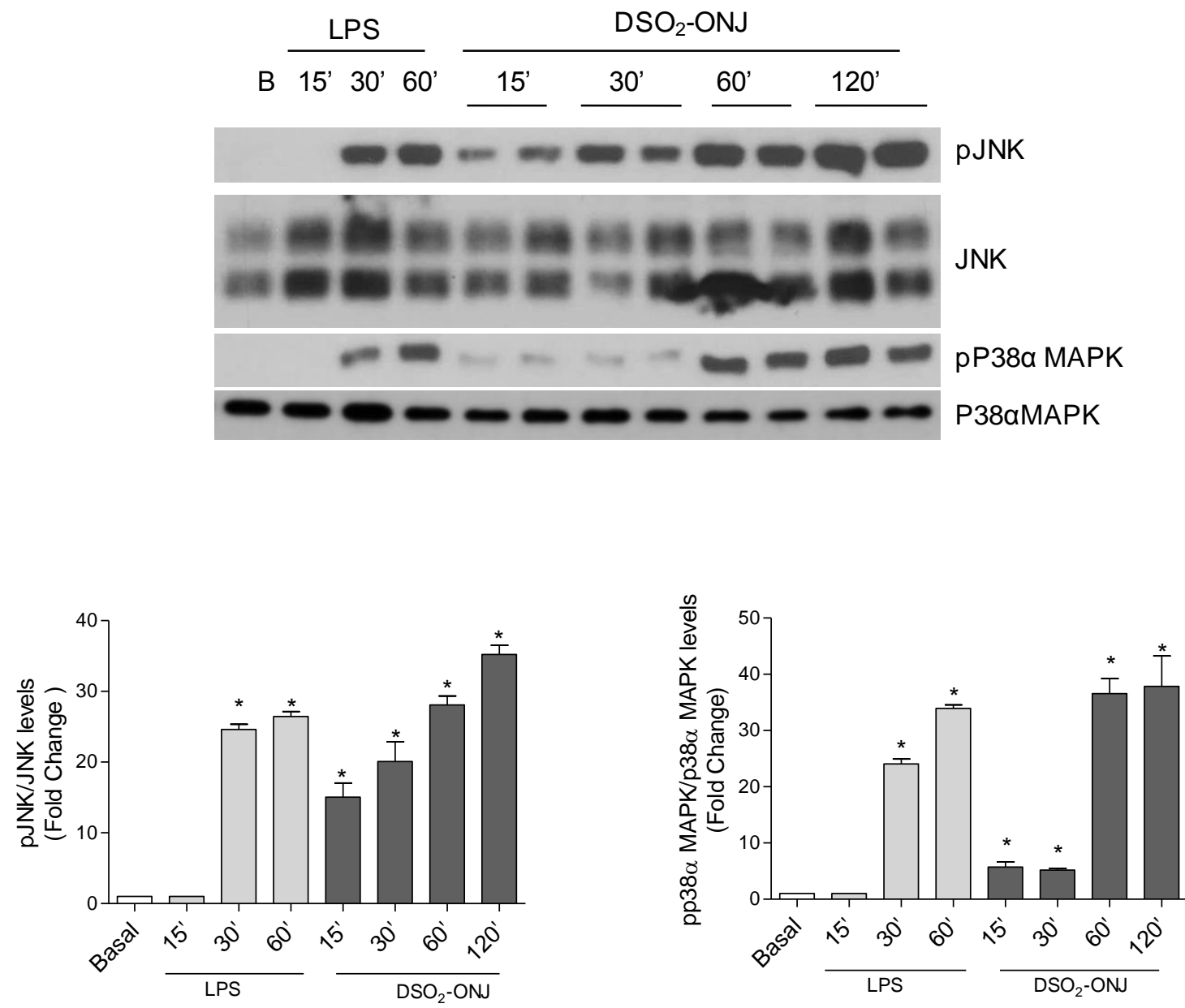
Figure 4. Direct effect of $\mathrm{DSO}_{2}-\mathrm{ONJ}$ in the activation of the MAPKs JNK and p38a

\subsection{Involvement of $\mathrm{p38} \alpha \mathrm{MAPK}$ in the effects of $\mathrm{DSO}_{2}-\mathrm{ONJ}$ in $\mathrm{Bv} .2$ microglial cells.}

As shown in Figure 1E, $\mathrm{DSO}_{2}-\mathrm{ONJ}$ reduces the effect of LPS on the induction of iNOS protein levels. As expected, Bv.2 microglial cells treated with the combination of LPS and the p38 $\alpha$ MAPK inhibitor SB203580 showed a substantial reduction of iNOS protein expression (Figure 5). Interestingly, protein levels of iNOS were further reduced by the combination of LPS+SB203580+DSO $2-\mathrm{ONJ}$, reflecting a synergistic effect of SB203580 and $\mathrm{DSO}_{2}-\mathrm{ONJ}$. 


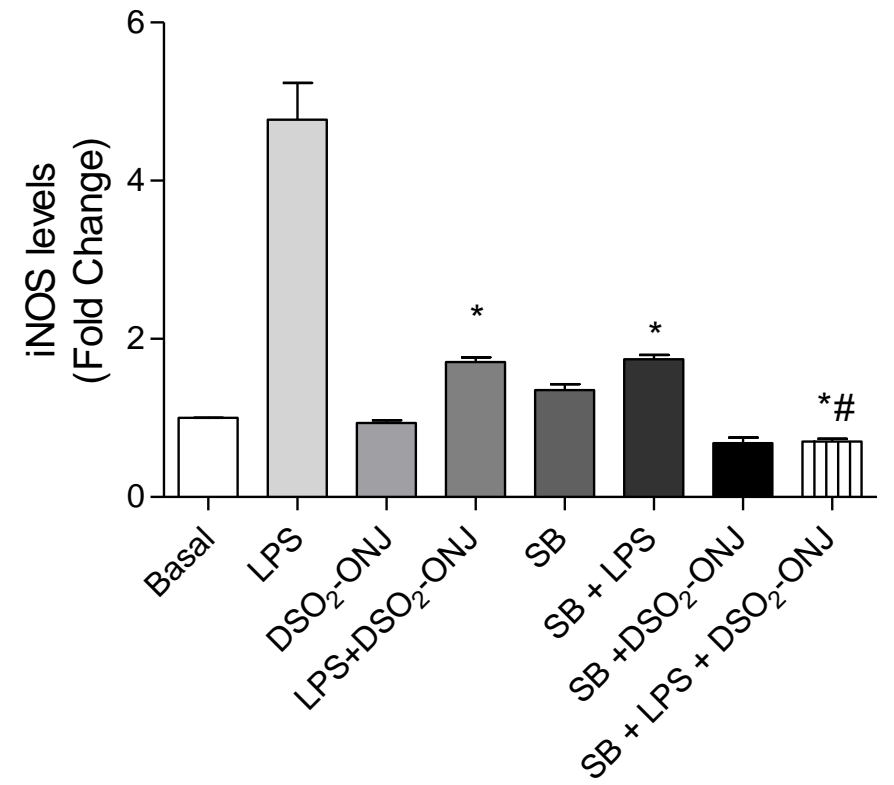

Figure 5. Effects of the p38a MAPK inhibitor SB203580 in the modulation iNOS by LPS and $\mathrm{DSO}_{2}-\mathrm{ONJ}$ in Bv.2 microglial cells. Bv.2 microglial cells were pretreated for $30 \mathrm{~min}$ with SB203580, followed by stimulation with $200 \mathrm{ng} / \mathrm{ml}$ LPS and $10 \mu \mathrm{M} \mathrm{DSO} 2^{-}$ ONJ for $24 \mathrm{~h}$. The protein extracts were analyzed by Western blot with antibodies against iNOS and $\alpha$-Tubulin. Representative autoradiograms are shown $(n=5$ independent experiments). Blots were quantified by performing scanning densitometry, and the results are mean \pm S.E.M. The ratio between the two indicated proteins and the 
fold change relative to Basal condition is shown. ${ }^{*} p \leq 0.05$ vs LPS treatment. ${ }^{*} p \leq 0.05$ vs $\mathrm{LPS}+\mathrm{DSO}_{2}-\mathrm{ONJ}$ treatment. ${ }^{\dagger} \mathrm{P} \leq 0.05$ vs $\mathrm{LPS}+\mathrm{SB} 203580$ treatment (two-way ANOVA followed by Bonferroni t-test).

\subsection{HO-1 was induced by $\mathrm{DSO}_{2}-\mathrm{ONJ}$ in $\mathrm{Bv} .2$ microglial cells and retinal explants from $d b / d b$ mice.}

Previous studies have shown that HO-1 induction exerts protective effects in cells stimulated by pro-inflammatory cytokines through the down-regulation of inflammatory mediators [43, 44]. Based on these studies, we analyzed the mRNA levels of the antiinflammatory cytokine IL10. As shown in Figure 6A, LPS markedly decreased 1110 mRNA levels, an effect abolished in the presence of $\mathrm{DSO}_{2}$-ONJ. Next, we analyzed the protein expression of $\mathrm{HO}-1$ in $\mathrm{Bv} .2$ microglial cells stimulated with $\mathrm{DSO}_{2}$-ONJ in the presence and absence of LPS. As Figure 6B shows, $\mathrm{DSO}_{2}-\mathrm{ONJ}$ increased $\mathrm{HO}-1$ levels in microglial cells either alone or in the presence of LPS. However, compound $\mathrm{DSO}_{2}$ ONJ did not counteract the effect of LPS on arg-1 mRNA levels (encoding arginase-1), a marker of M2 polarization stage of microglia (Figure 6C). 
A
B
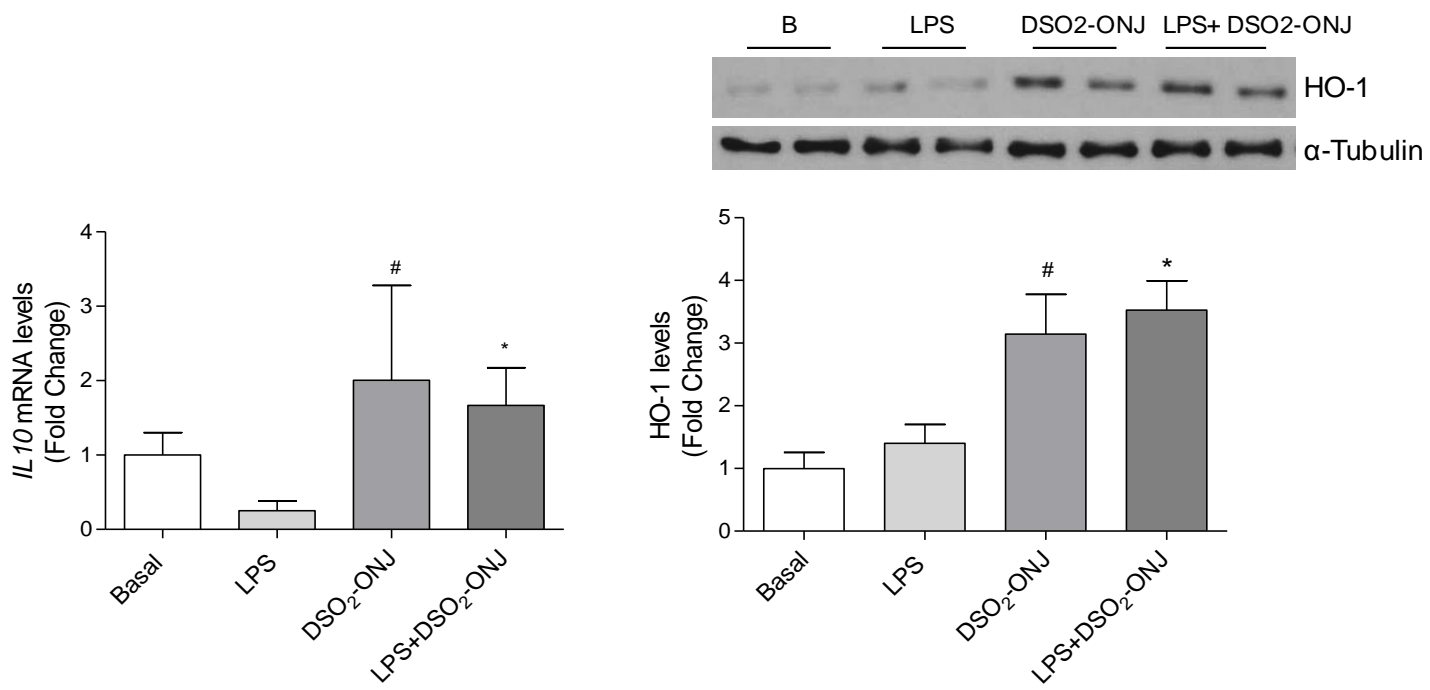

C

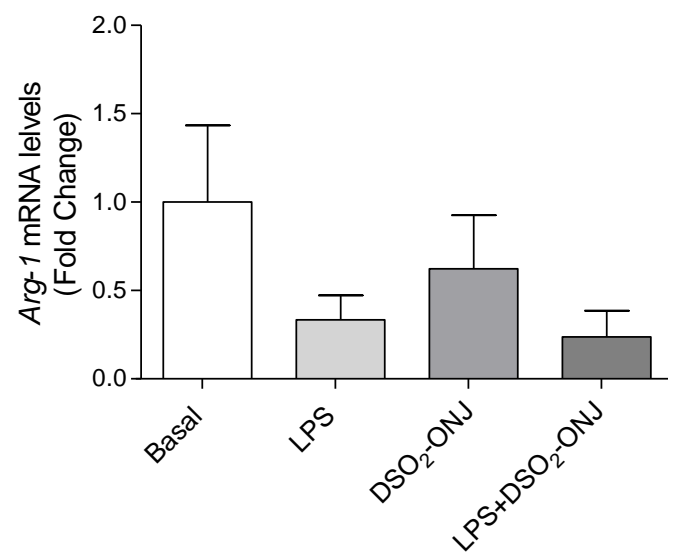

Figure 6. Induction of an anti-inflammatory response mediated by $\mathrm{HO}-1$ and IL-10 in Bv.2 microglial cells treated with $\mathrm{DSO}_{2}$-ONJ. Bv.2 microglial cells were stimulated with $200 \mathrm{ng} / \mathrm{ml}$ LPS and $10 \mu \mathrm{M} \mathrm{DSO} 2^{-O N J}$ for $24 \mathrm{~h}$. A) Ill0 mRNA levels were determined by RT-PCR. The results are means \pm S.E.M. $(n=5$ independent experiments). The fold change levels relative to Basal condition is shown. ${ }^{*} p \leq 0.05$ vs LPS treatment; \# $p \leq 0.05$ vs Basal treatment. B) Protein extracts were analyzed by Western blot with antibodies against $\mathrm{HO}-1$ and $\alpha$-Tubulin. Representative autoradiograms are shown ( $n=5$ independent experiments). Blots were quantified by performing scanning densitometry and the results are mean \pm S.E.M. The fold change of 
$H O-1$ levels relative to Basal condition is shown. ${ }^{*} p \leq 0.05$ vs LPS treatment; \# $p \leq 0.05$ vs Basal treatment. C) Arg-1 mRNA levels were determined by RT-PCR. The results are means \pm S.E.M. ( $n=5$ independent experiments $).$

We next analyzed the effect of the $\mathrm{sp}^{2}-\mathrm{IGL} \mathrm{DSO}_{2}-\mathrm{ONJ}$ in whole retinal explants from diabetic $d b / d b$ mice that presented established DR at the age of 8 weeks [7, 8]. Retinal explants from 8 weeks-old $d b /+$ control and $d b / d b$ mice were treated for $24 \mathrm{~h}$ with $\mathrm{DSO}_{2}-\mathrm{ONJ}$ and the expression of HO-1 was evaluated by Western blot. As shown in Figure 7A, treatment with $\mathrm{DSO}_{2}-\mathrm{ONJ}$ increased $\mathrm{HO}-1$ expression exclusively in retinal explants from $d b / d b$ mice. Also, the expression of iNOS, which was elevated in retinal explants from $d b / d b$ mice, was significantly reduced by the treatment with $\mathrm{DSO}_{2}-\mathrm{ONJ}$ compound (Figure 7B).
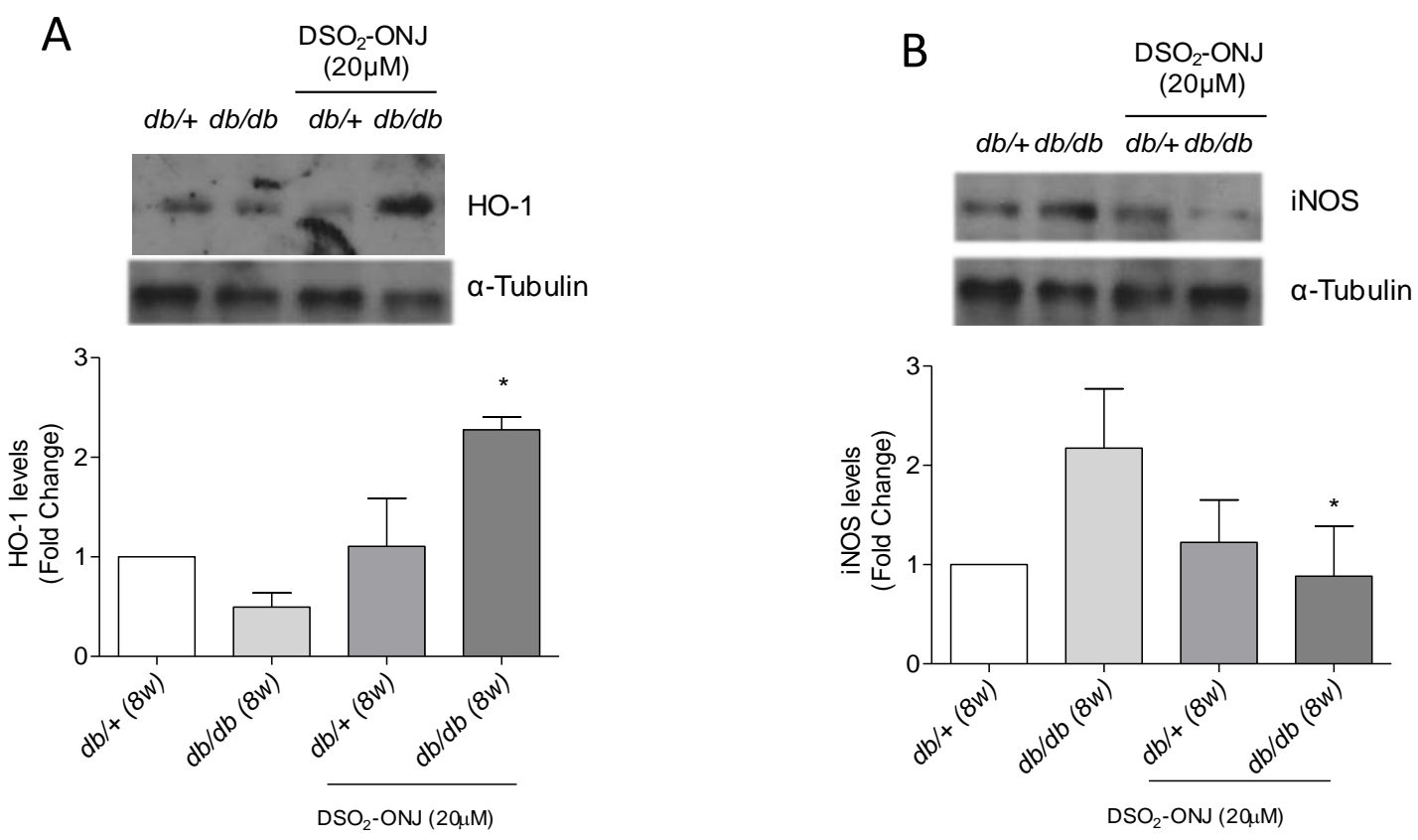
Figure 7. Treatment of retinal explants from $\mathrm{db} / \mathrm{db}$ mice with $\mathrm{DSO}_{2}-\mathrm{ONJ}$ compound decreased iNOS, increased $\mathrm{HO}-1$ and reduced reactive gliosis. Retinal explants from 8 weeks old $d b / d b$ and $d b /+$ mice were treated for $24 h$ with $D_{S O}-O N J(20 \mu M)$ or vehicle. Western blot was performed with the corresponding antibodies against $\mathrm{HO}-1$ (A) or iNOS (B) using $\alpha$-Tubulin as loading control. Blots were quantified by performing scanning densitometry and the results are mean \pm S.E.M. The fold change levels relative to Basal condition is shown. ${ }^{*} p \leq 0.05$ vs $d b / d b$ Representative autoradiograms are shown ( $n=5$ retinas per condition). C) Retinal explants from $\mathrm{db} / \mathrm{db}$ mice at 8 weeks of age were treated for $24 \mathrm{~h}$ with $\mathrm{DSO}_{2}-\mathrm{ONJ}(20 \mu \mathrm{M})$ (right panel) or with vehicle (left panel). Immunostaining for GFAP (green) was carried out in whole retinas. Representative images are shown ( $n=5$ retinas per condition).

3.6. Retinal gliosis is reduced by the treatment of retinal explants from $d b / d b$ mice with $\mathrm{DSO}_{2}-\mathrm{ONJ}$. 
To get more insights in the benefit of increasing HO-1 levels in the whole retina by administration of $\mathrm{DSO}_{2}-\mathrm{ONJ}$ under a pro-inflammatory environment we evaluated the reactive gliosis, which is a hallmark of neurodegenerative diseases of the retina, including DR [45, 46]. We have previously shown that reactive gliosis is already present in the retinas from obese/diabetic $d b / d b$ mice at 8 weeks of age, and that this effect was maintained in retinal explants [47]. To achieve this, retinal explants from 8 weeks-old $d b / d b$ mice were treated with $\mathrm{DSO}_{2}-\mathrm{ONJ}$ for $24 \mathrm{~h}$ and subsequently analyzed by immunofluorescence with the anti-GFAP antibody. As shown in Figure 7C, reactive gliosis was totally blunted in retinal explants from $d b / d b$ mice treated with $\mathrm{DSO}_{2}-\mathrm{ONJ}$ compound at $20 \mu \mathrm{M}$ for $24 \mathrm{~h}$.

\subsection{Docking of $\mathrm{DSO}_{2}-\mathrm{ONJ}$ into p38a MAPK}

The overall topology of $\mathrm{p} 38 \alpha$ MAPK consists of two lobes (N' and C') that form a groove in between hosting the catalytic site (Figure 8A) [48]. The available crystal structures of $\mathrm{p} 38 \alpha$ MAPK in complex with PIAs and perifosine show that these lipid molecules bind at a hydrophobic pocket located at the C'-lobe distal from the active site [45]. Upon binding, they induce local conformational changes in the $\alpha E F / \alpha F$ loop region (residues 194-200), leading to p38 $\alpha$ MAPK autophosphorylation and activation. The similar chemical structure of $\mathrm{sp}^{2}$-IGLs and these activating lipid molecules led to the hypothesis that $\mathrm{DSO}_{2}$-ONJ might bind at the same site in the kinase C'-lobe. To probe this notion, we performed docking experiments between $\mathrm{DSO}_{2}-\mathrm{ONJ}$ and $\mathrm{p} 38 \alpha$ MAPK. Two main binding modes, with similar estimated binding free energies, have been obtained for the binding of $\mathrm{DSO}_{2}$-ONJ into this hydrophobic pocket (Figure 8). As observed in the 4E6A and 4E6C crystallographic complexes, the polar regions of $\mathrm{DSO}_{2}$ ONJ are predicted to form only few polar interactions with some main-chain groups. In 
the first complex (Figure 8B), these polar regions are more solvent exposed. In the second one (Figure $8 \mathrm{C}$ ), $\mathrm{DSO}_{2}-\mathrm{ONJ}$ binds in a mode reminiscent to that observed for PIA23 in the crystallographic complex (Supplementary Figure 2). These results are consistent with the prediction, supporting that $\mathrm{DSO}_{2}-\mathrm{ONJ}$ elicit $\mathrm{p} 38 \alpha$ MAPK autophosphorylation and activation through a mechanism analogous to that evidenced for PIAs or perifosine.

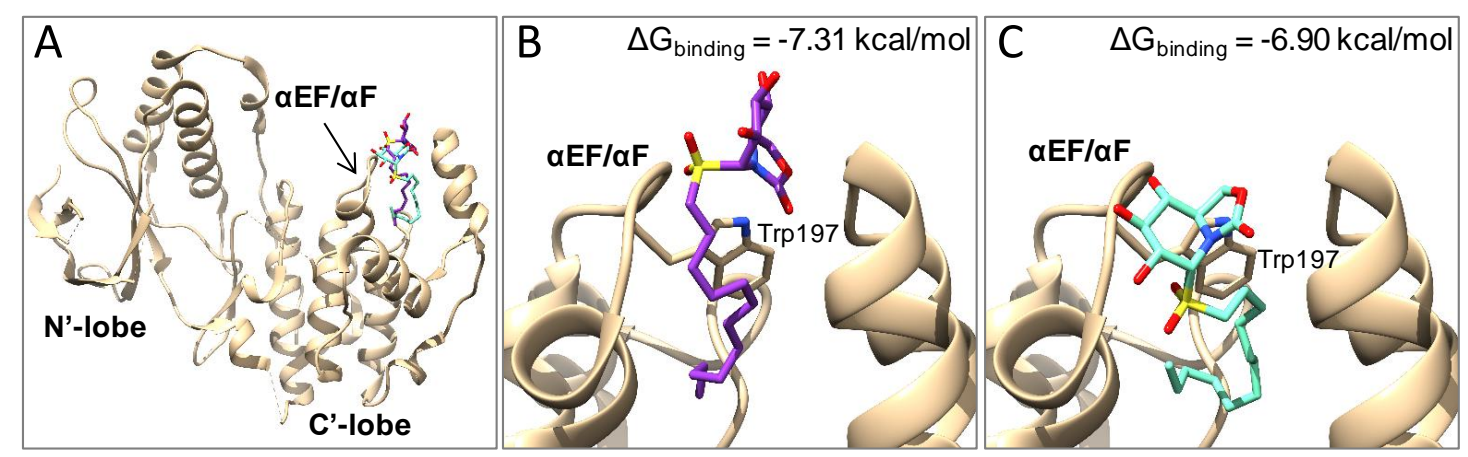

Figure 8. Predicted binding of $\mathrm{DSO}_{2}-\mathrm{ONJ}$ into p38a MAPK. Docking calculations of $D_{S O}-O N J$ into the $p 38 \alpha M A P K$ structure from the PDB ID 4E6A predict two main binding modes, with similar estimated binding free energies $\left(\Delta G_{\text {binding }}\right)$. A) Overall view of p38a MAPK (beige cartoon) with the two predicted binding modes for $\mathrm{DSO}_{2}$-ONJ shown. The ligand is represented in sticks and coloured by element with purple and cyan carbon atoms for the first and second binding modes, respectively. Hydrogen atoms are not represented for clarity. $\mathrm{B}$ ) and $\mathrm{C}$ ) are closer views to the $\mathrm{DSO}_{2}-\mathrm{ONJ}$ first and second binding modes, respectively. Trp197 is shown in beige sticks.

\section{DISCUSSION}

Diabetic retinopathy has an important pro-inflammatory component associated with alterations in the immune responses, including the dynamics of microglia polarization 
[8]. In fact, targeting neuroinflammation by modulating the polarization state of microglia has been suggested as a therapeutic strategy to delay and/or prevent the deterioration of visual function in diabetic patients [49].

Several types of lipid molecules, including PIAs or the alkyl ether lipids (e.g. perifosine) and their glycosydated derivatives, which were initially developed as antitumor agents, have been found to display anti-inflammatory properties $[8,50,51]$. $\mathrm{sp}^{2}$-Iminosugar glycolipids ( $\mathrm{sp}^{2}$-IGLs) represent a recent addition to this list. Although the mechanisms involved in their biological activities are still poorly understood, the structural similarities with the PIAs and their interference with the same biochemical routes suggest that $\mathrm{sp}^{2}$-IGLs and PIAs may share the same targets [51]. In the context of DR, we have recently reported the anti-inflammatory effect of 1R-DSO-ONJ, a member of the $\mathrm{sp}^{2}$-IGL family, by targeting pro-inflammatory signaling in microglia [8]. In the present study, we have investigated whether $\mathrm{DSO}_{2}$-ONJ [35], a close analogue incorporating a sulfone group that better mimic the phosphate group in PIAs, exerts anti-inflammatory effects counteracting LPS in Bv.2 microglial cells, and also in an ex vivo model of retinal explants from diabetic $d b / d b$ mice. A step further, we have conducted research on the molecular mechanisms involved.

After an initial screening of the dose-dependent effect of $\mathrm{DSO}_{2}-\mathrm{ONJ}$ in the cellular viability of Bv.2 microglial cells, we found that at non-toxic concentrations (1-10 $\mu \mathrm{M})$ this compound was able to inhibit NO production induced by LPS-stimulation, one of the key features of the inflammatory stages. Regarding the molecular mechanisms by which $\mathrm{DSO}_{2}$-ONJ elicits the anti-inflammatory effects in microglial cells, it almost completely blocked LPS-induced Nos2 mRNA, iNOS protein levels, as well as decreased mRNA levels of pro-inflammatory cytokines while, simultaneously, it increased the mRNA levels of the anti-inflammatory cytokine IL10. The analysis of 
classical pro-inflammatory signaling cascades revealed that $\mathrm{DSO}_{2}-\mathrm{ONJ}$ efficiently inhibited LPS-mediated degradation of I $\mathrm{B}$ and the translocation of $\mathrm{p} 65 \mathrm{NF} \kappa \mathrm{B}$ to the nucleus, as well as the NLRP3 inflammasome complex by targeting Nlrp3 mRNA and IL1 $\beta$ processing by caspase-1. However, $\mathrm{DSO}_{2}-\mathrm{ONJ}$ failed to inhibit JNK or $\mathrm{p} 38 \alpha$ MAPK phosphorylation and, more importantly, the latter kinases were directly activated by $\mathrm{DSO}_{2}-\mathrm{ONJ}$.

It is well known that the phosphorylation of JNK and p38 $\alpha$ MAPK, key molecules of the MAPKs signaling pathway, is critical for the ability of immune cells to respond to various pro-inflammatory stimuli including LPS [53]. As stated above, $\mathrm{DSO}_{2}-\mathrm{ONJ}$ was able to induce the activation of both MAPKs in the same way that LPS. In the light of these results, it has been shown that compounds such as chitosan and its derivatives elicit anti-inflammatory effects in macrophages through the activation of both MAPKs [54], and other studies have reported anti-inflammatory effects mediated by specific activation of p38 $\alpha$ MAPK [18]. Although the canonical signaling mediated by upstream MAPK kinases (MKKs) is considered to be the main pathway for MAPKs activation, several alternative mechanisms for the activation of $\mathrm{p} 38 \alpha$ MAPK isoform have been reported [55]. Interestingly, PIAs have been shown to concomitantly induce p38 $\alpha$ MAPK autophosphorylation and activation in a MKK3/6 dependent and independent manner [19], and a similar p38 $\alpha$ MAPK activation effect has been reported for perifosine [20]. Given the similar chemical nature of $\mathrm{DSO}_{2}-\mathrm{ONJ}$ and these activating lipid molecules, we hypothesized that they may all bind at the same site in p38 $\alpha$ MAPK. The structures of $\mathrm{p} 38 \alpha$ MAPK in complex with PIAS or perifosine revealed similar mode of binding at a novel hydrophobic binding site in the kinase $\mathrm{C}^{\prime}$-lobe formed in part by the p38 $\alpha$ MAPK insert region and also identified local and significant conformational changes of the $\alpha \mathrm{EF} / \alpha \mathrm{F}$ loop that could be the basis of the autoactivation 
properties [39]. Importantly, this loop, located in the vicinity of the activation loop, has no sequence and conformational similarity within the MAPKs family or to other kinases. In this regard, it is reasonable to speculate that $\mathrm{DSO}_{2}-\mathrm{ONJ}$ might also act as an intracellular inductor of $\mathrm{p} 38 \alpha$ MAPK phosphorylation by binding at the same site in the protein as PIAs or perifosine, thereby activating its downstream signaling pathways. Docking experiments support this hypothesis, suggesting even a more favourable binding energy as compared with PIAs or perifosine (Figure 8 and Supplementary Figures 2-4). Additional experimental evidence for this alternative p38 $\alpha$ MAPK activation by $\mathrm{DSO}_{2}-\mathrm{ONJ}$ is supported by the fact that in the presence of SB203580, an inhibitor of the classical activation of $\mathrm{p} 38 \alpha$ MAPK by dual phosphorylation, $\mathrm{DSO}_{2}-\mathrm{ONJ}$ was still able to maintain p38 $\alpha$ MAPK-mediated anti-inflammatory effects in LPSstimulated Bv.2 cells.

We next addressed the mechanism by which the direct activation of p38 $\alpha$ MAPK by $\mathrm{DSO}_{2}-\mathrm{ONJ}$ could be involved in its anti-inflammatory effects in microglial cells. Activation of $\mathrm{p} 38 \alpha$ MAPK has been shown to induce HO-1 expression in various cell types including macrophages [46], hepatocytes [47], pulmonary epithelial cells [48], and vascular cells [49]. HO-1 is an important anti-oxidant molecule and its upregulation is a critical cytoprotective mechanism activated during cellular stress [56, 57]. In this work we have demonstrated that in Bv.2 microglial cells there is a direct relationship between the induction of $\mathrm{HO}-1$ protein expression and elevation of $\mathrm{IllO}$ mRNA by the treatment with the $\mathrm{sp}^{2}$-iminosugar glycolipid $\mathrm{DSO}_{2}-\mathrm{ONJ}$. It is important to note that HO-1 and IL-10 can perpetuate their anti-inflammatory effects in a reciprocal manner [49]. Moreover, both HO-1-induced IL-10 production and IL-10induced HO-1 activation are dependent on p38 $\alpha$ MAPK activation [50-53]. In this regard our results strongly suggest that $\mathrm{DSO}_{2}-\mathrm{ONJ}$ selectively up-regulates the synthesis 
of the anti-inflammatory cytokine IL-10 in a manner likely dependent on HO-1 due to both the canonical and non-canonical p38 $\alpha$ MAPK activation [58].

Our previous data in retinal explants from $d b / d b$ mice at 8 weeks of age have shown that treatment with $1 \mathrm{R}-\mathrm{DSO}-\mathrm{ONJ}$, another $\mathrm{sp}^{2}$-IGL representative, ameliorated reactive gliosis [8]. A step further, the results presented herein revealed that iNOS expression, which was elevated in the retina of diabetic $d b / d b$ mice, was reduced after treatment of retinal explants with $\mathrm{DSO}_{2}-\mathrm{ONJ}$ that in parallel increased $\mathrm{HO}-1$ levels, reinforcing the link between inflammation and $\mathrm{HO}-1$ in the retina. Interestingly, reactive gliosis in retinal explants from $d b / d b$ mice was also reduced by the treatment with $\mathrm{DSO}_{2}-\mathrm{ONJ}$. As reactive gliosis is present in $\mathrm{DR}[7,58,59]$, our results might have therapeutic implications proposing a novel strategy for its treatment based on the specific induction of $\mathrm{HO}-1$ in the retina.

In conclusion and as summarized in Figure 9, the results of this study have demonstrated a protective effect of $\mathrm{DSO}_{2}-\mathrm{ONJ}$ compound in Bv.2 microglial cells and in retinal explants from $d b / d b$ mice that, in addition of targeting NFkB and the inflammasome, can be mediated through the direct activation of p38 $\alpha$ MAPK. This latter effect leads to the induction of HO-1 and IL-10, boosting the anti-inflammatory efficacy of this compound. Therefore, the induction of HO-1 and its associated antiinflammatory mechanisms could strengthen the structural and immunological protection of the retina during inflammatory processes such those occurring in DR. 
Figure 9. Schematic representation of the signaling pathways involved in the antiinflammatory effects of $\mathrm{DSO}_{2}-\mathrm{ONJ}$ in microglial cells. $\mathrm{DSO}_{2}-\mathrm{ONJ}$ compound elicits beneficial effects in microglia during neuroinflammation associated to DR by blocking the pro-inflammatory $N F \kappa B$ pathway. Additionally, $\mathrm{DSO}_{2}$-ONJ activates $p 38 \alpha \mathrm{MAPK}$ which, in turn, induces HO-1 and ILIO and confers anti-oxidant and anti-inflammatory protection in the retina. 


\section{Contributions}

AIA researched data and wrote and reviewed the manuscript. AMV provided funding, wrote and reviewed the manuscript. EA-E researched data. EMSF researched data. COM provided funding and researched data. JMGF provided funding and researched data. LM provided funding and conducted the computational experiments. AMV and AIA are responsible for the integrity of the work as a whole.

\section{Duality of interest}

The authors declare that there is no duality of interest associated with this manuscript.

\section{Acknowledgments}

We acknowledge Drs Lucía Sánchez-Ruiloba, and Diego Navarro (IIB Alberto Sols, CSIC, Madrid, Spain) for assistance with microscopy. This work was supported by grants from the Spanish Ministry of Economy and Competitiveness: SAF2015-65267-R (MINECO/FEDER), SAF2016-76083-R (MINECO-FEDER), CTQ2015-64425-C2-1-R (MINECO-FEDER) and CTQ2014-53144-P and grants from the Spanish ISCIII (CIBERdem) and INFLAMES (ISCIII PIE14/00045, co-funded by ERDF, “Investing in your future").

\section{BIBLIOGRAPHY}

1. Saltiel, A.R. and J.M. Olefsky, Inflammatory mechanisms linking obesity and metabolic disease. J Clin Invest, 2017. 127(1): p. 1-4.

2. Becher, B., S. Spath, and J. Goverman, Cytokine networks in neuroinflammation. Nat Rev Immunol, 2017. 17(1): p. 49-59.

3. Yau, J.W., et al., Global prevalence and major risk factors of diabetic retinopathy. Diabetes Care, 2012. 35(3): p. 556-64.

4. Tang, J. and T.S. Kern, Inflammation in diabetic retinopathy. Prog Retin Eye Res, 2011. 30(5): p. 343-58.

5. Zeng, H.Y., W.R. Green, and M.O. Tso, Microglial activation in human diabetic retinopathy. Arch Ophthalmol, 2008. 126(2): p. 227-32. 
6. Krady, J.K., et al., Minocycline reduces proinflammatory cytokine expression, microglial

activation, and caspase-3 activation in a rodent model of diabetic retinopathy. Diabetes, 2005. 54(5): p. 1559-65.

7. Bogdanov, P., et al., The $d b / d b$ mouse: a useful model for the study of diabetic retinal neurodegeneration. PLoS One, 2014. 9(5): p. e97302.

8. Arroba, A.I., et al., Modulation of microglia polarization dynamics during diabetic retinopathy in $d b / d b$ mice. Biochim Biophys Acta, 2016. 1862(9): p. 1663-74.

9. Demircan, N., et al., Determination of vitreous interleukin-1 (IL-1) and tumour necrosis factor (TNF) levels in proliferative diabetic retinopathy. Eye (Lond), 2006. 20(12): p. 1366-9.

10. Hernandez, C., et al., Interleukin-8, monocyte chemoattractant protein-1 and IL-10 in the vitreous fluid of patients with proliferative diabetic retinopathy. Diabet Med, 2005. 22(6): p. 719-22.

11. Baynes, J.W., Role of oxidative stress in development of complications in diabetes. Diabetes, 1991. 40(4): p. 405-12.

12. Calderon, G.D., et al., Oxidative stress and diabetic retinopathy: development and treatment. Eye (Lond), 2017.

13. Kowluru, R.A. and M. Mishra, Epigenetic regulation of redox signaling in diabetic retinopathy: Role of Nrf2. Free Radic Biol Med, 2017. 103: p. 155-164.

14. Gao, L. and G.E. Mann, Vascular NAD(P)H oxidase activation in diabetes: a double-edged sword in redox signalling. Cardiovasc Res, 2009. 82(1): p. 9-20.

15. Kang, K.W., et al., Phosphatidylinositol 3-kinase regulates nuclear translocation of NF-E2-related factor 2 through actin rearrangement in response to oxidative stress. Mol Pharmacol, 2002. 62(5): p. 1001-10.

16. Shyong, M.P., et al., Viral delivery of heme oxygenase-1 attenuates photoreceptor apoptosis in an experimental model of retinal detachment. Vision Res, 2008. 48(22): p. 2394-402.

17. He, M., et al., Roles for redox signaling by NADPH oxidase in hyperglycemia-induced heme oxygenase-1 expression in the diabetic retina. Invest Ophthalmol Vis Sci, 2013. 54(6): p. 4092101.

18. Paine, A., et al., Signaling to heme oxygenase-1 and its anti-inflammatory therapeutic potential. Biochem Pharmacol, 2010. 80(12): p. 1895-903.

19. Gills, J.J., et al., Phosphatidylinositol ether lipid analogues that inhibit AKT also independently activate the stress kinase, p38alpha, through MKK3/6-independent and -dependent mechanisms. J Biol Chem, 2007. 282(37): p. 27020-9.

20. Gills, J.J. and P.A. Dennis, Perifosine: update on a novel Akt inhibitor. Curr Oncol Rep, 2009. 11(2): p. 102-10.

21. Force, T. and K.L. Kolaja, Cardiotoxicity of kinase inhibitors: the prediction and translation of preclinical models to clinical outcomes. Nat Rev Drug Discov, 2011. 10(2): p. 111-26.

22. Jaffres, P.A., et al., Alkyl ether lipids, ion channels and lipid raft reorganization in cancer therapy. Pharmacol Ther, 2016. 165: p. 114-31.

23. Ceylan-Isik, A.F., et al., Herbal and traditional Chinese medicine for the treatment of cardiovascular complications in diabetes mellitus. Curr Diabetes Rev, 2008. 4(4): p. 320-8.

24. Mendonca, P., et al., The attenuating effects of 1,2,3,4,6 penta-O-galloyl-beta-d-glucose on inflammatory cytokines release from activated BV-2 microglial cells. J Neuroimmunol, 2017. 305: p. 9-15.

25. Wrodnigg, T.M., A.J. Steiner, and B.J. Ueberbacher, Natural and synthetic iminosugars as carbohydrate processing enzyme inhibitors for cancer therapy. Anticancer Agents Med Chem, 2008. 8(1): p. 77-85.

26. Martin, O., [Iminosugars: current and future therapeutic applications]. Ann Pharm Fr, 2007. 65(1): p. 5-13.

27. Durantel, D., C. Alotte, and F. Zoulim, Glucosidase inhibitors as antiviral agents for hepatitis $B$ and C. Curr Opin Investig Drugs, 2007. 8(2): p. 125-9.

28. Greimel, P., et al., Iminosugars and relatives as antiviral and potential anti-infective agents. Curr Top Med Chem, 2003. 3(5): p. 513-23.

29. Wrodnigg, T.M. and F.K. Sprenger, Bioactive carbohydrates and recently discovered analogues as chemotherapeutics. Mini Rev Med Chem, 2004. 4(4): p. 437-59. 
30. Mitrakou, A., et al., Long-term effectiveness of a new alpha-glucosidase inhibitor (BAY m1099-

31. Scott, L.J. and C.M. Spencer, Miglitol: a review of its therapeutic potential in type 2 diabetes mellitus. Drugs, 2000. 59(3): p. 521-49.

32. Sanchez-Fernandez, E.M., et al., Glycomimetic-based pharmacological chaperones for lysosomal storage disorders: lessons from Gaucher, GM1-gangliosidosis and Fabry diseases. Chem Commun (Camb), 2016. 52(32): p. 5497-515.

33. Mena-Barragan, T., et al., pH-Responsive Pharmacological Chaperones for Rescuing Mutant Glycosidases. Angew Chem Int Ed Engl, 2015. 54(40): p. 11696-700.

34. Allan, G., et al., New castanospermine glycoside analogues inhibit breast cancer cell proliferation and induce apoptosis without affecting normal cells. PLoS One, 2013. 8(10): p. e76411.

35. Sánchez-Fernández, E. M.; et al., Antileishmanial activity of $\mathrm{sp}^{2}$-iminosugar derivatives. RSC Adv. 2015, 5, 21812-21822

36. Díaz-Pérez, P., et al., Synthesis and comparative glycosidase inhibitory properties of reducing castanospermine analogues. Eur. J. Org. Chem. 2005. 2903-2913.

37. Green, L.C., et al., Analysis of nitrate, nitrite, and [15N]nitrate in biological fluids. Anal Biochem, 1982. 126(1): p. 131-8.

38. Morris, G.M., et al., AutoDock4 and AutoDockTools4: Automated docking with selective receptor flexibility. J Comput Chem, 2009. 30(16): p. 2785-91.

39. Tzarum, N., et al., Lipid molecules induce p38alpha activation via a novel molecular switch. J Mol Biol, 2012. 424(5): p. 339-53.

40. Dolinsky, T.J., et al., PDB2PQR: an automated pipeline for the setup of Poisson-Boltzmann electrostatics calculations. Nucleic Acids Res, 2004. 32(Web Server issue): p. W665-7.

41. Pettersen, E.F., et al., UCSF Chimera--a visualization system for exploratory research and analysis. J Comput Chem, 2004. 25(13): p. 1605-12.

42. Boutagy, N.E., et al., Metabolic endotoxemia with obesity: Is it real and is it relevant? Biochimie, 2016. 124: p. 11-20.

43. Guillen, M.I., et al., The CO-releasing molecule CORM-2 is a novel regulator of the inflammatory process in osteoarthritic chondrocytes. Rheumatology (Oxford), 2008. 47(9): p. 1323-8.

44. Megias, J., et al., Heme oxygenase-1 induction modulates microsomal prostaglandin E synthase1 expression and prostaglandin E(2) production in osteoarthritic chondrocytes. Biochem Pharmacol, 2009. 77(12): p. 1806-13.

45. Vohra, R., J.C. Tsai, and M. Kolko, The role of inflammation in the pathogenesis of glaucoma. Surv Ophthalmol, 2013. 58(4): p. 311-20.

46. Buschini, E., et al., Age related macular degeneration and drusen: neuroinflammation in the retina. Prog Neurobiol, 2011. 95(1): p. 14-25.

47. Arroba, A.I. and A.M. Valverde, Inhibition of Protein Tyrosine Phosphatase 1B Improves IGF-I Receptor Signaling and Protects Against Inflammation-Induced Gliosis in the Retina. Invest Ophthalmol Vis Sci, 2015. 56(13): p. 8031-44.

48. Wilson, K.P., et al., Crystal structure of p38 mitogen-activated protein kinase. J Biol Chem, 1996. 271(44): p. 27696-700.

49. Arroba, A.I. and A.M. Valverde, Modulation of microglia in the retina: new insights into diabetic retinopathy. Acta Diabetol, 2017. 54(6): p. 527-533.

50. Gradziel, C.S., et al., d-3-Deoxy-dioctanoylphosphatidylinositol induces cytotoxicity in human MCF-7 breast cancer cells via a mechanism that involves downregulation of the D-type cyclinretinoblastoma pathway. Biochim Biophys Acta, 2016. 1861(11): p. 1808-1815.

51. Semini, G., et al., Inositol-C2-PAF down-regulates components of the antigen presentation machinery in a 2D-model of epidermal inflammation. Biochem Pharmacol, 2014. 87(3): p. 47788.

52. Gueder, N., et al., sp2 -Iminosugar alpha-glucosidase inhibitor 1-C-octyl-2-oxa-3oxocastanospermine specifically affected breast cancer cell migration through Stim1, beta1integrin, and FAK signaling pathways. J Cell Physiol, 2017.

53. Guha, M. and N. Mackman, LPS induction of gene expression in human monocytes. Cell Signal, 2001. 13(2): p. 85-94. 
54. Hyung, J.H., et al., Involvement of Nrf2-mediated heme oxygenase-1 expression in antiinflammatory action of chitosan oligosaccharides through MAPK activation in murine macrophages. Eur J Pharmacol, 2016. 793: p. 43-48.

55. Lee, J.C., et al., A protein kinase involved in the regulation of inflammatory cytokine biosynthesis. Nature, 1994. 372(6508): p. 739-46.

56. Choi, A.M. and J. Alam, Heme oxygenase-1: function, regulation, and implication of a novel stress-inducible protein in oxidant-induced lung injury. Am J Respir Cell Mol Biol, 1996. 15(1): p. 9-19.

57. Bauer, M. and I. Bauer, Heme oxygenase-1: redox regulation and role in the hepatic response to oxidative stress. Antioxid Redox Signal, 2002. 4(5): p. 749-58.

58. Carrasco, E., et al., Lower somatostatin expression is an early event in diabetic retinopathy and is associated with retinal neurodegeneration. Diabetes Care, 2007. 30(11): p. 2902-8.

59. Biswal, M.R., et al., A hypoxia-responsive glial cell-specific gene therapy vector for targeting retinal neovascularization. Invest Ophthalmol Vis Sci, 2014. 55(12): p. 8044-53. 


\section{SUPPLEMENTARY FIGURES}
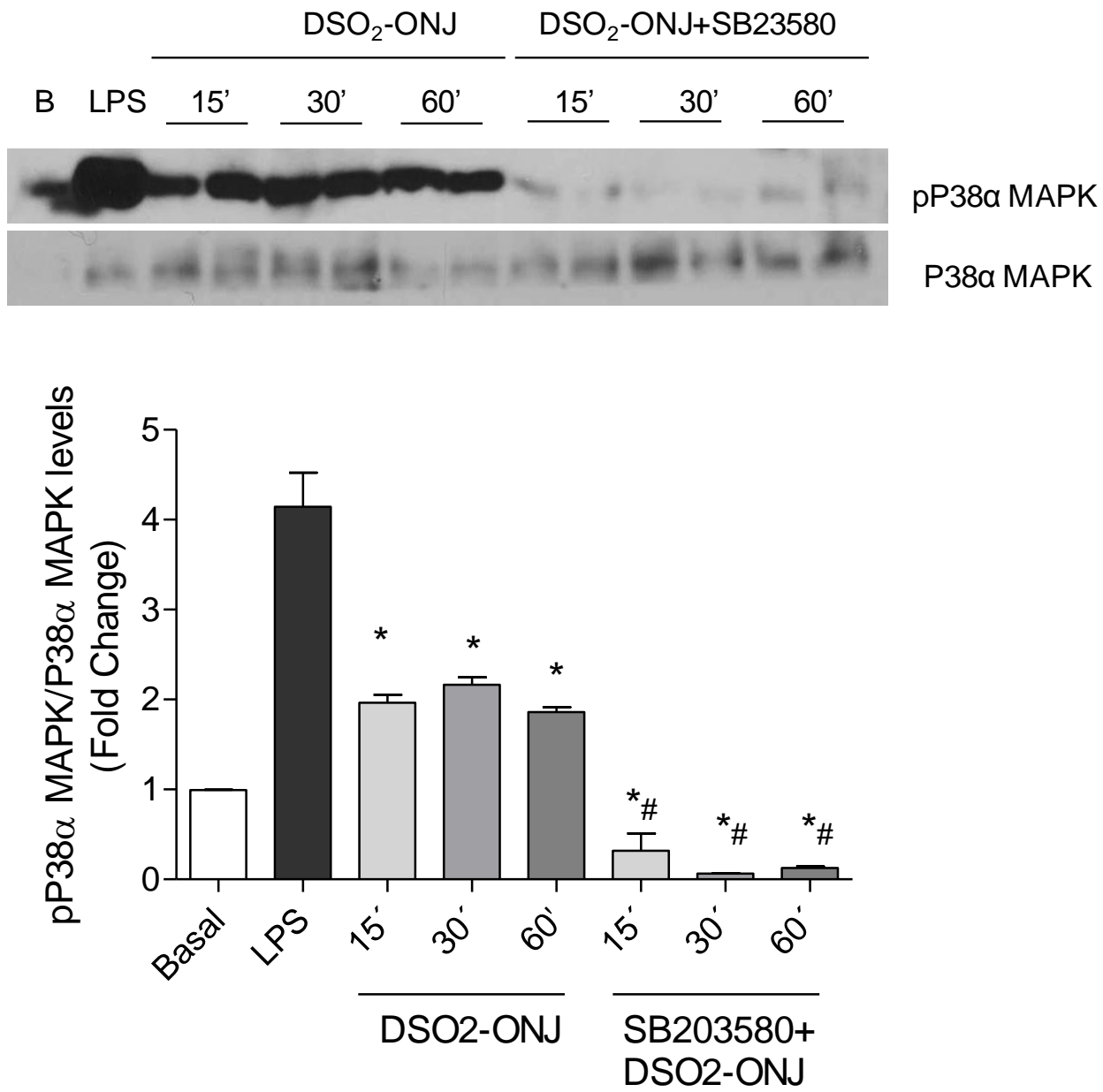

Figure Supplementary 1. $\mathrm{DSO}_{2}$-ONJ-induced p38a MAPK phosphorylation is totally abolished by the inhibitor SB203580. Bv.2 microglial cells were pretreated for $30 \mathrm{~min}$ with SB203580, followed by stimulation with $200 \mathrm{ng} / \mathrm{ml}$ LPS or $10 \mu \mathrm{M} \mathrm{DSO} \mathrm{S}_{2}$-ONJ for the indicated time periods. The protein extracts were analyzed by Western blot with

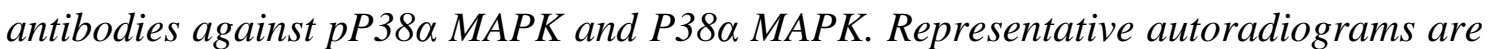
shown ( $n=5$ independent experiments). Blots were quantified by performing scanning densitometry, and the results are mean \pm S.E.M. The ratio between the two indicated proteins and the fold change relative to Basal condition is shown. ${ }^{*} \leq 0.05$ vs Basal treatment. ${ }^{\#} \leq 0.05$ vs $\mathrm{DSO}_{2}-\mathrm{ONJ}$ treatment. (two-way ANOVA followed by Bonferroni $t$ test). 


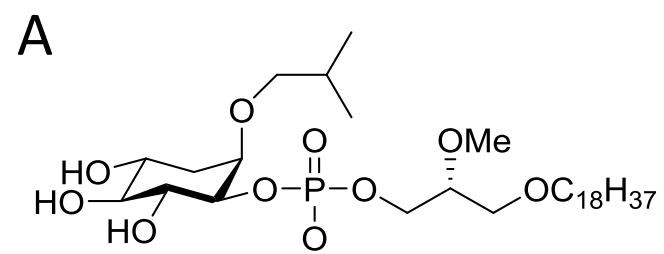

PIA13
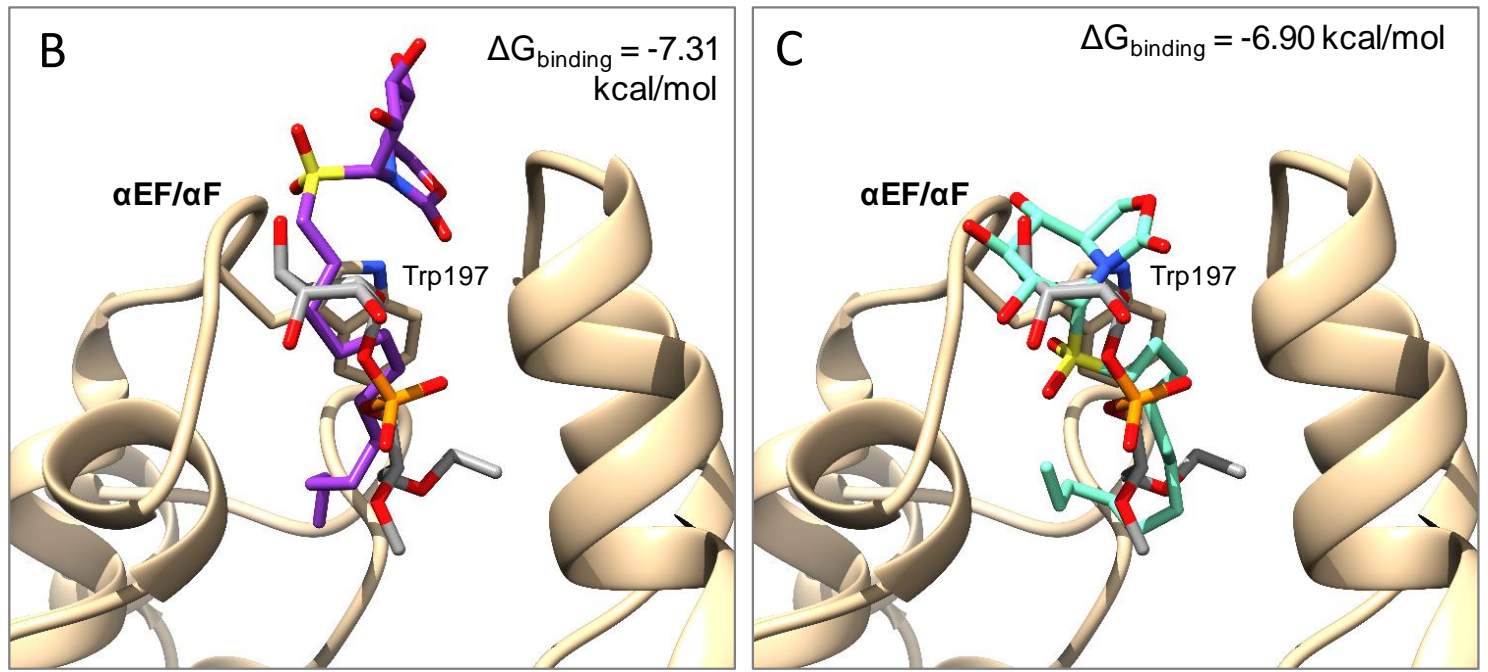

Figure Supplementary 2. Comparison of the predicted binding of $\mathrm{DSO}_{2}-\mathrm{ONJ}$ to $\mathrm{p38 \alpha}$ MAPK (coordinates from PDB ID 4E6A) with that observed for PIA23 in the crystallographic complex PDB ID 4E6A. Docking calculations of $\mathrm{DSO}_{2}$-ONJ into the p38a structure from the PDB ID 4E6A predicted two main binding modes, with similar estimated binding free energies $\left(\Delta G_{\text {binding }}\right)$. The ligands are represented in sticks and coloured by element with gray, purple or cyan carbon atoms for PIA23 (A) and the first and second binding modes of $\mathrm{DSO}_{2}-\mathrm{ONJ}$, respectively. Hydrogen atoms are not represented for clarity. Trp197 is shown in beige sticks. In the first complex $(\boldsymbol{B})$, the polar regions of $\mathrm{DSO}_{2}-\mathrm{ONJ}$ are more solvent exposed. In the second one $(\mathrm{C}), \mathrm{DSO}_{2}$ ONJ adopts a binding orientation more similar to that observed for PIA23. 


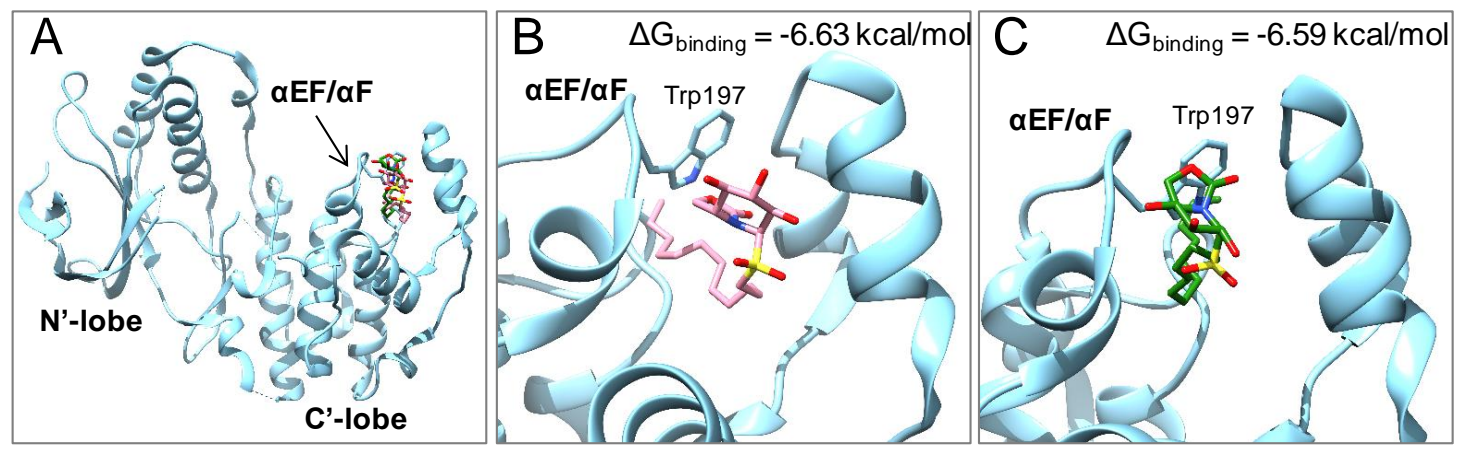

Figure S3. Predicted binding of $\mathrm{DSO}_{2}-\mathrm{ONJ}$ to $\mathrm{p38 \alpha} \mathrm{MAPK}$ using the protein coordinates from the PDB ID 4E6C. Two best docking solutions for the binding of $D_{S O} O_{2}$-ONJ into the p38a structure from the PDB ID 4E6C (based on the estimated binding free energies, $\Delta G_{\text {binding }}$. The binding affinity is predicted to be better in the $4 E 6 A$ structure. A) Overall view of p38a MAPK (blue cartoon) with the two predicted binding modes shown. The ligand is represented in sticks and coloured by element with pink and green carbon atoms for the first and second binding modes, respectively. Hydrogen atoms are not represented for clarity. $\boldsymbol{B})$ and $\boldsymbol{C}$ ) are closer views to the $\mathrm{DSO}_{2}$-ONJ first and second binding modes, respectively. Trp197 is shown in blue sticks as a reference. 
A
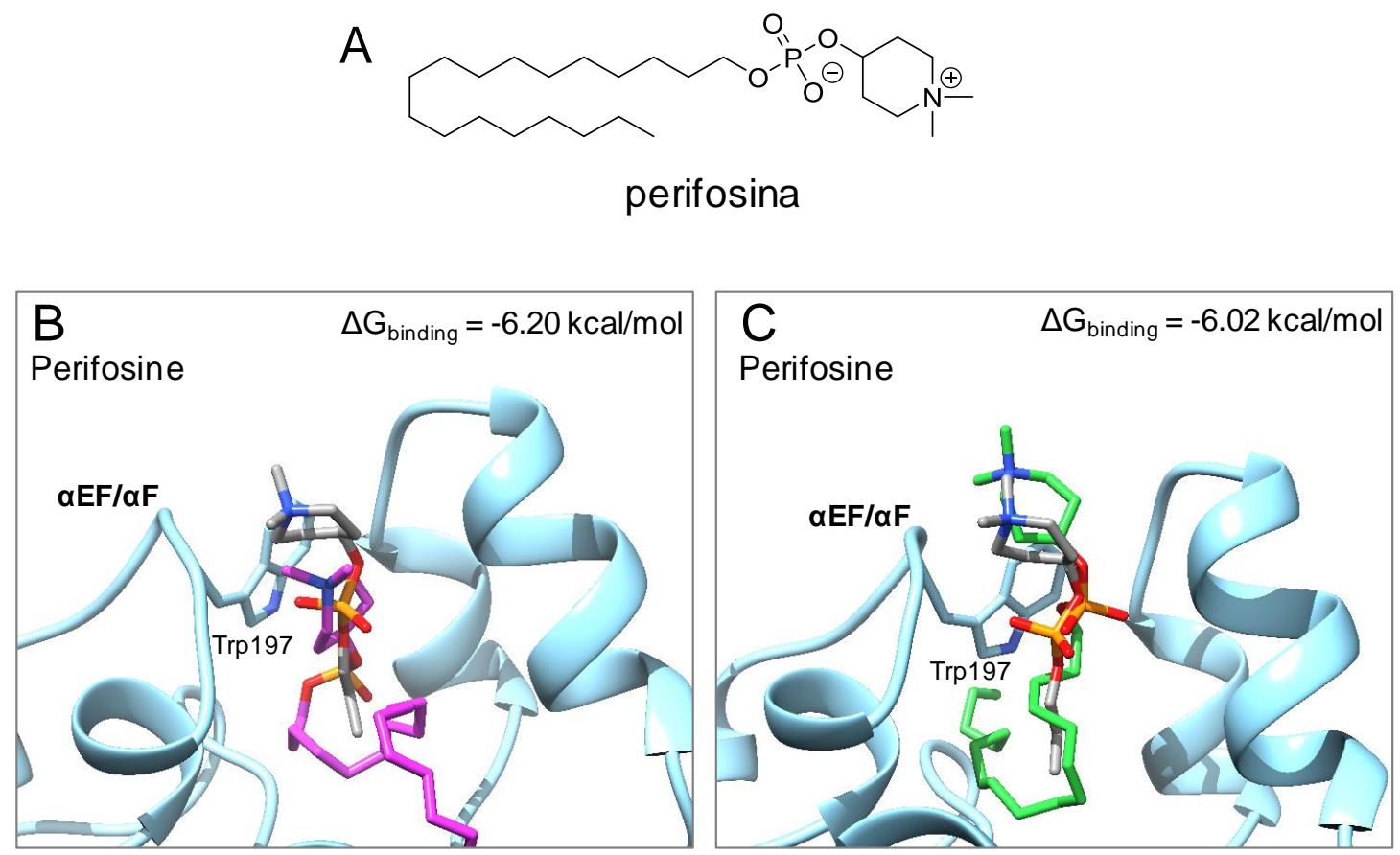

Figure S4. Docking of perifosine into p38a MAPK (coordinates from PDB ID 4E6C) and comparison with its crystallographic complex (PDB ID 4E6C). The two best binding modes obtained in the calculations (based on the estimated binding free energies, $\left.\Delta G_{\text {binding }}\right)$, are shown superimposed with the crystallographic structure. Both complexes present similar binding free energies, being the second one more similar to the crystallographic structure. Perifosine $(\boldsymbol{A})$ is represented in sticks and coloured by element with gray, magenta or green carbon atoms for the crystallographic structure, the first $(\boldsymbol{B})$ and second $(\boldsymbol{C})$ calculated binding modes, respectively. Hydrogen atoms are not represented for clarity. The protein is shown in blue cartoon and Trp197 is represented in blue sticks as a reference. 


\section{Please wait...}

If this message is not eventually replaced by the proper contents of the document, your PDF viewer may not be able to display this type of document.

You can upgrade to the latest version of Adobe Reader for Windows®, Mac, or Linux® by visiting http://www.adobe.com/go/reader_download.

For more assistance with Adobe Reader visit http://www.adobe.com/go/acrreader.

Windows is either a registered trademark or a trademark of Microsoft Corporation in the United States and/or other countries. Mac is a trademark of Apple Inc., registered in the United States and other countries. Linux is the registered trademark of Linus Torvalds in the U.S. and other countries. 


\section{Please wait...}

If this message is not eventually replaced by the proper contents of the document, your PDF viewer may not be able to display this type of document.

You can upgrade to the latest version of Adobe Reader for Windows®, Mac, or Linux® by visiting http://www.adobe.com/go/reader_download.

For more assistance with Adobe Reader visit http://www.adobe.com/go/acrreader.

Windows is either a registered trademark or a trademark of Microsoft Corporation in the United States and/or other countries. Mac is a trademark of Apple Inc., registered in the United States and other countries. Linux is the registered trademark of Linus Torvalds in the U.S. and other countries. 


\section{Please wait...}

If this message is not eventually replaced by the proper contents of the document, your PDF viewer may not be able to display this type of document.

You can upgrade to the latest version of Adobe Reader for Windows®, Mac, or Linux® by visiting http://www.adobe.com/go/reader_download.

For more assistance with Adobe Reader visit http://www.adobe.com/go/acrreader.

Windows is either a registered trademark or a trademark of Microsoft Corporation in the United States and/or other countries. Mac is a trademark of Apple Inc., registered in the United States and other countries. Linux is the registered trademark of Linus Torvalds in the U.S. and other countries. 


\section{Please wait...}

If this message is not eventually replaced by the proper contents of the document, your PDF viewer may not be able to display this type of document.

You can upgrade to the latest version of Adobe Reader for Windows ${ }^{\circledR}$, Mac, or Linux ${ }^{\circledR}$ by visiting http://www.adobe.com/products/acrobat/readstep2.html.

For more assistance with Adobe Reader visit http://www.adobe.com/support/products/ acrreader.html.

Windows is either a registered trademark or a trademark of Microsoft Corporation in the United States and/or other countries. Mac is a trademark of Apple Inc., registered in the United States and other countries. Linux is the registered trademark of Linus Torvalds in the U.S. and other countries. 


\section{Please wait...}

If this message is not eventually replaced by the proper contents of the document, your PDF viewer may not be able to display this type of document.

You can upgrade to the latest version of Adobe Reader for Windows®, Mac, or Linux® by visiting http://www.adobe.com/go/reader_download.

For more assistance with Adobe Reader visit http://www.adobe.com/go/acrreader.

Windows is either a registered trademark or a trademark of Microsoft Corporation in the United States and/or other countries. Mac is a trademark of Apple Inc., registered in the United States and other countries. Linux is the registered trademark of Linus Torvalds in the U.S. and other countries. 
To view the full contents of this document, you need a later version of the PDF viewer. You can upgrade to the latest version of Adobe Reader from www.adobe.com/products/acrobat/readstep2.html

For further support, go to www.adobe.com/support/products/acrreader.html 


\section{Please wait...}

If this message is not eventually replaced by the proper contents of the document, your PDF viewer may not be able to display this type of document.

You can upgrade to the latest version of Adobe Reader for Windows®, Mac, or Linux® by visiting http://www.adobe.com/go/reader_download.

For more assistance with Adobe Reader visit http://www.adobe.com/go/acrreader.

Windows is either a registered trademark or a trademark of Microsoft Corporation in the United States and/or other countries. Mac is a trademark of Apple Inc., registered in the United States and other countries. Linux is the registered trademark of Linus Torvalds in the U.S. and other countries. 